\title{
Multi-period Defined Contribution Pension Funds Investment Management with Regime-Switching and Mortality risk ${ }^{\text {th }}$
}

\author{
Haixiang $\mathrm{Yao}^{\mathrm{a}}$, Ping Chen ${ }^{\mathrm{b}}$, Xun $\mathrm{Li}^{\mathrm{c}, *}$ \\ ${ }^{a}$ School of Finance, Guangdong University of Foreign Studies, Guangzhou 510006, China \\ ${ }^{b}$ Department of Economics, The University of Melbourne, Parkville, Victoria 3010, Australia \\ ${ }^{c}$ Department of Applied Mathematics, The Hong Kong Polytechnic University, Hong Kong
}

\begin{abstract}
Using mean-variance criterion, we investigate a multi-period defined contribution pension fund investment problem in a Markovian regime-switching market. Both stochastic wage income and mortality risk are incorporated in our model. In a regime-switching market, the market mode changes among a finite number of regimes, and the market state process is modeled by a Markov chain. The key parameters, such as the bank interest rate, or expected returns and covariance matrix of stocks, will change according to the market state. By virtue of Lagrange duality technique, dynamic programming approach and matrix representation method, we derive expressions of efficient investment strategy and its efficient frontier in closed-form. Also, we study some special cases of our model. Finally, a numerical example based on real data from the American market sheds light on our theoretical results.
\end{abstract}

Keywords: Contribution pension funds; Multi-period mean-variance; Regime switching; Mortality risk; Dynamic programming

\section{Introduction}

There are two major types of pension schemes: defined benefit (DB) and defined contribution (DC). In a DB plan, benefits are fixed in advance by sponsors, and contributions are set and subsequently adjusted so as to ensure that the fund remains in balance, hence the risk is borne by sponsors. It is borne by members in the

\footnotetext{
This research is supported by grants from the National Natural Science Foundation of China (No. 71471045), the China Postdoctoral Science Foundation (No. 2014M560658, 2015T80896), and the Characteristic and Innovation Foundation of Guangdong Colleges and Universities (Humanity and Social Science Type), and the Hong Kong RGC grants 15209614 and 15224215.

*Corresponding author. Tel.: +852 27666939.

Email addresses: yaohaixiang@gdufs.edu.cn (Haixiang Yao), pche@unimelb.edu.au (Ping Chen), malixun@polyu.edu.hk (Xun Li)
} 
case of DC plan, where contributions are predefined (often as a fixed percentage of wage income) and benefits are affected by investment performance and wage income of the member during the accumulation phase. Nowadays, DC pension funds play an important role in the social security system all over the world. Note that an accumulated fund may not be adequate on the day of retirement, hence it is essential for the member to have investments during the accumulation phase. Optimal DC fund investments have important applications in various fields including, but not limited to, economics, finance and actuarial science. Recently, there has been increasing interest in studying this type of stochastic problem as well as addressing its applications.

Based on the mean square error minimization (quadratic target-based), Haberman and Vigna (2002) investigate an investment problem of multi-period DC pension funds during the accumulation phase. They obtain optimal investment strategies by using dynamic programming approach. Using the expected utility maximization, Deelstra et al. (2003) and Giacinto et al. (2011) derive optimal asset allocation for DC pension funds with stochastic interest rates and a minimum guarantee protection. Deelstra et al. (2004) further study optimal design of the minimum guarantee by virtue of martingale method. Under the framework of continuous-time utility maximization, Han and Hung (2012) gain optimal asset allocation for DC pension funds with stochastic income and inflation risk. Based on the target-driven criterion and loss aversion framework (prospect theory), Blake et al. (2013) post an optimal asset allocation problem for DC pension funds. He and Liang (2015) study optimal asset allocation and benefit outgo policies of DC pension fund, and derive the closedform solutions of optimal policies using HJB and variational inequality methods. For more detailed discussions on this topic, we refer to Cairns et al. (2006), Gao (2009) and Emms et al. (2012), Guan and Liang (2014), and Konicz and Mulvey (2015).

The aforementioned works suppose that there is only one market state with deterministic coefficients. In the real world, however, various market states reflect random nature of the underlying market environment. In recent years, regime-switching models have become popular for reflecting various states of financial market. Generally, in a regime-switching market, the market mode can take values in one of a finite number of regimes, and the market state process is modeled by a Markov chain. The key parameters, such as the bank interest rate, or stocks appreciation and volatility rates, will change according to the market state.

Parameter estimation for regime-switching models in economics can date back to Quandt (1958) who attempts to estimate parameters for linear regression under two regimes. Hamilton (1989) introduces a filter algorithm in his fitting of regime- 
switching models to the data of post war US real GNP data. The estimated results of different regimes fit the actual business cycle remarkably well, which set a milestone in the development of regime-switching models. Empolying maximum likelihood with relatively minor modifications to the recursive filter in Hamilton (1989), Kim et al. (2008) further study the estimation of Markov regime-switching regression models with endogenous switching. With respect to data with fat tails, Eichler and Tuerk (2013) propose a base regime in which data is log-normally distributed and a spike regime where the form of distribution is not predetermined. Then, a semiparametric approach, which provides a better fit for data with non-lognormal distribution like energy prices, is applied to estimate the marginal distribution function and parameters of the spike regime.

Regarding the number of regimes (market states), there are some empirical works. For example, in Hamilton and Susmel (1994), several regime-switching models are analyzed, varying the number of regimes and the form of the model within regimes. In their fitting of the various weekly econometric series, they find out that the more complicated autoregressive conditional heteroskedastic (ARCH)-type models with regimes seem to be necessary. Hardy (2001) uses monthly data from the Standard \& Poor's 500 (S \& P) and the Toronto Stock Exchange 300 (TSE) indices to fit a regime switching lognormal model. She investigates two- and three-regime models and describes that there is no significant improvement in fit for the TSE data set from adding the third regime, and only a marginal improvement for the S \& P data set. Also, Sims and Zha (2006) apply regime-switching models with different regimes to fit the U.S. monetary policy data, and show that more than two regimes should be used to capture the dynamics better. Hence, the number of regimes depends heavily on the type and origin of data.

General portfolio selection problems with regime-switching have been considered in many papers. For example, using a multi-period utility maximization model, Cheung and Yang (2007) and Çanakoglu and Özekici (2010) study the optimal investment-consumption and the optimal investment-only strategy, respectively. Based on continuous-time utility maximization criterion, Yiu et al. (2010) investigate the optimal portfolios with a Value-at-Risk constraint; Shen and Siu (2012) consider the optimal asset allocation with stochastic interest rate; Liu (2011) studies an optimal consumption and portfolio choice problem under the ambiguity and regime-switching mean returns. However, the application of regime-switching models on DC pension funds investment management problems is underexplored. Problems with these features have been studied in only very few papers: maximizing the expected utility of the terminal wealth (see Korn et al. (2011)); minimizing the risk 
described by a convex risk measure (see Siu (2012)).

Except for regime-switching market modes, mortality risk is also important for life insurance and pension fund management. Yaari (1965), Richard (1975) and Pliska and Ye (2007) analyze an optimal consumption-investment problem with uncertain lifetime in the case of life insurance. Based on the expected utility maximization, the optimal dividend and asset allocation with mortality risk of a DB pension fund is considered by Hainaut and Devolder (2007). By minimizing the risk which is a quadratic target-based cost function, Hainaut and Deelstra (2011) investigate the optimal contribution rate of a DB pension fund with stochastic mortality which is modeled by a jump process.

In recent years, mean-variance criteria, which was pioneered by Markowitz (1952) and extended to dynamic setting by Li and Ng (2000) and Zhou and Li (2000)), have been applied to investment management of pension funds. In a continuoustime setting, Delong et al. (2008) and Josa-Fombellida and Rincón-Zapatero (2008) study the optimal investment and contribution strategies for DB pension funds; Yao et al. (2013), Vigna (2014) and Guan and Liang (2015) investigate the optimal investment strategies for DC pension fund. Yao et al. (2014) study an optimal asset allocation for a DC pension fund with stochastic income and mortality risk within the multi-period mean-variance framework. Hu and Zeng (2015) and Wu et al. (2015) consider multi-period and continuous-time equilibrium investment strategies for DC pension schemes under the mean-variance criterion, respectively.

In this paper, we further develop the work of Yao et al. (2014) to study regimeswitching market. Using a multi-period mean-variance model, we investigate an investment management problem for DC pension funds with regime-switching market states. Both stochastic income and mortality risk are incorporated. These extensions are not only of great interest in theory, but also of practical importance. From a mathematical point of view, the inclusion of regime-switching, stochastic income and mortality risk makes the problem harder in tackling the resulting Bellman equation by the dynamic programming approach. The computation is more complicated to obtain the closed form solutions. We will adopt the stochastic optimal control method and Lagrange dual theory in our calculation.

The remainder of the paper is organized as follows. Section 2 outlines the general model. By introducing a Lagrange multiplier, Section 3 transforms the original model into a standard multi-period stochastic linear quadratic control problem. The corresponding analytical solutions are derived by the dynamic programming approach. In Section 4, the expressions for the efficient investment strategy and the mean-variance efficient frontier are obtained by the Lagrange dual theory. Some 
special cases are discussed in Section 5. Section 6 presents some numerical results of our model and Section 7 concludes.

\section{Model formulation}

Throughout the paper, let the market state at time $k$ for $k=0,1, \cdots, T$ be denoted by $\xi_{k} \in \Pi$, where $\Pi=\{1,2, \cdots, m\}$. Suppose that the state process $\left\{\xi_{k}\right\}$ follows a Markov chain, and its transition probability matrix is $\mathbb{Q}(k)=\left(q_{i j}(k)\right)_{m \times m}$, where $q_{i j}(k)=\operatorname{Pr}\left(\xi_{k+1}=j \mid \xi_{k}=i\right)$ satisfying $\sum_{j=1}^{m} q_{i j}(k)=1$ for $i=1,2, \cdots, m$, where $\operatorname{Pr}(\cdot)$ is probability measure. Consider a representative wage earner who enters the pension plan at time 0 with an initial fund $x_{0}(\geq 0)$ in his/her account and initial wage income $y_{0}(>0)$. Upon the retirement, the wage earner can convert his/her pension fund into an annuity such that he/she can receive a scheduled pension stream in the following years. If the wage earner dies before the retirement, then his/her family can withdraw all the money which has been contributed into the pension fund.

Denote by $x_{k}$ and $y_{k}$ the wealth of the pension fund and the wage income of the wage earner at time $k$, respectively, for $k=0,1, \cdots, T$. Suppose that the wage income is stochastic and follows the dynamics

$$
y_{k+1}=b_{k}\left(\xi_{k}\right) y_{k}, k=0,1, \cdots, T-1,
$$

where $b_{k}\left(\xi_{k}\right)$ is a random variable with its probability distribution depending on the market state $\xi_{k}$, and represents the stochastic growth rate of the wage income over period $k$ (from time $k$ to time $k+1$ ). Note that the wage income cannot be negative, we assume that $b_{k}\left(\xi_{k}\right)>0$ almost surely for $k=0,1, \cdots, T-1$. Suppose that the wage earner contributes a fixed percentage $c$ of his/her wage income at the beginning of each period until his/her retirement at time $T$, where $c$ is a constant and called contribution rate. Namely, $c y_{k}$ is the amount of contribution at time $k$, then just after this contribution at time $k$, the wealth of the pension fund is $x_{k}+c y_{k}$.

Remark 1. In order to make our model more general, we don't need to assume $c>0$. When $c=0$, we have $c y_{k}=0$, then our model degenerates to an ordinary multi-period mean-variance portfolio selection problem. When $c<0$, then $c y_{k}$ can be interpreted as the distribution of the pension fund over period $k$ for $k=$ $0,1, \cdots, T-1$. Therefore, our model can also be used to study the DC pension fund management problem in the de-cumulation phase. 
Assume that the financial market consisting of $N+1$ assets can include or exclude a risk free asset, and the random returns of these assets all depend on the market state during any time period. Denote by $\boldsymbol{e}_{k}\left(\xi_{k}\right)=\left(e_{k}^{0}\left(\xi_{k}\right), e_{k}^{1}\left(\xi_{k}\right), \cdots, e_{k}^{N}\left(\xi_{k}\right)\right)^{\prime}$ the random return vector of these $N+1$ assets over the period $k(k=0,1, \cdots, T-$ 1) with their probability distributions depending on the market state $\xi_{k}$, where ' represents the transpose of a matrix or a vector. Suppose that the pension fund can be invested in the $N+1$ assets in the market. Denote by $u_{k}^{n}$ the amount invested in the $n$th asset over period $k$ for $n=1,2, \cdots, N$ and $k=1,2, \cdots, T$. Then incorporating the contribution $c y_{k}$ at the beginning of period $k$, the amount invested in the 0 th asset over period $k$ is $\left(x_{k}+c y_{k}\right)-\sum_{n=1}^{N} u_{k}^{n}$ for $k=1,2, \cdots, T$. Therefore, the wealth process $x_{k}$ of the pension fund follows the dynamics

$$
\begin{aligned}
x_{k+1} & =e_{k}^{0}\left(\xi_{k}\right)\left(\left(x_{k}+c y_{k}\right)-\sum_{n=1}^{N} u_{k}^{n}\right)+\sum_{n=1}^{N} e_{k}^{n}\left(\xi_{k}\right) u_{k}^{n} \\
& =x_{k} e_{k}^{0}\left(\xi_{k}\right)+c y_{k} e_{k}^{0}\left(\xi_{k}\right)+\boldsymbol{P}_{k}^{\prime}\left(\xi_{k}\right) \boldsymbol{u}_{k},
\end{aligned}
$$

where

$$
\boldsymbol{P}_{k}\left(\xi_{k}\right)=\left(e_{k}^{1}\left(\xi_{k}\right)-e_{k}^{0}\left(\xi_{k}\right), e_{k}^{2}\left(\xi_{k}\right)-e_{k}^{0}\left(\xi_{k}\right), \cdots, e_{k}^{N}\left(\xi_{k}\right)-e_{k}^{0}\left(\xi_{k}\right)\right)^{\prime}, \boldsymbol{u}_{k}=\left(u_{k}^{1}, u_{k}^{2}, \cdots, u_{k}^{N}\right)^{\prime} .
$$

The wage earner plans to retire at time $T$, however, in reality, he may die and has to terminate the pension fund plan before the retired time $T$ due to the mortality risk (e.g., traffic accident or serious illness, etc.). We assume that the wage earner is alive at time $t=0$ and has a lifetime (the time of death) denoted by $\tau$, a positive random variable. If the death occurs during the $(k-1)$ th time period, i.e., $k-1<\tau \leq k$ and $k \leq T-1$, then the actual terminated time $T^{\tau}$ of the pension fund plan is $k$; if the death occurs after $T-1$, i.e., $\tau>T-1$, the actual terminated time $T^{\tau}=T$. That is

$$
T^{\tau}= \begin{cases}k, & k-1<\tau \leq k \text { and } k \leq T-1, \\ T, & \tau>T-1\end{cases}
$$

We now proceed on the choosing of the probability (mass) function of $T^{\tau}$. Let $S(t)=$ $\operatorname{Pr}(\tau \geq t \mid \tau>0)$ denote the survival probability of the wage earner. Following, Charupat Milevsky (2002), Pliska and Ye (2007)) and Yao et al. (2014), the survival probability $S(t)$ is given by $S(t)=e^{-\int_{0}^{t} \lambda(s) d s}$, where $\lambda(s)$ is the instantaneous hazard rate (mortality intensity) on $[0, t]$. By $(4)$, the probability mass function of $T^{\tau}$ is defined as

$$
p_{k}:=\operatorname{Pr}\left(T^{\tau}=k\right)= \begin{cases}S(k-1)-S(k), & k=1, \cdots, T-1, \\ S(T-1), & k=T .\end{cases}
$$


Then, it follows that

$$
p_{k}= \begin{cases}e^{-\int_{0}^{k-1} \lambda(s) d s}-e^{-\int_{0}^{k} \lambda(s) d s}, & k=1, \cdots, T-1, \\ e^{-\int_{0}^{T-1} \lambda(s) d s}>0, & k=T .\end{cases}
$$

Let $\wp_{k}$ be the $\sigma$-field representing the information available till time $k$. We call the investment strategy $\mathbb{u}=\left\{\boldsymbol{u}_{k} ; k=0,1, \cdots, T-1\right\}$ admissible if $\boldsymbol{u}_{k}$ is measurable to $\wp_{k}$. Let $\Theta_{k}$ denote the collection of all admissible strategies starting at time $k$. Let $\mathrm{E}[\cdot]=\mathrm{E}\left[\cdot \mid \wp_{0}\right]$ and $\operatorname{Var}[\cdot]=\operatorname{Var}\left[\left.\cdot\right|_{\wp_{0}}\right]$ be the expectation and variance operators under the condition of information set $\wp_{0}$, respectively. Following Chen and Yang (2011), and Costa and Oliveira (2012), in this paper, we have the assumptions as follows.

Assumption 1. $\mathrm{E}\left[\boldsymbol{e}_{k}(i) \boldsymbol{e}_{k}^{\prime}(i)\right]>0$. This means that $\mathrm{E}\left[\boldsymbol{e}_{k}(i) \boldsymbol{e}_{k}^{\prime}(i)\right]$ is a positive definite matrix for all $i \in \Pi$ and $k=0,1, \cdots, T-1$.

Assumption 1 means that assets are not redundant. We also assume that the future lifetime of the wage earner is independent of the financial market. That is,

Assumption 2. $\tau$ is statistically independent of $\left(\xi_{k}, b_{k}\left(\xi_{k}\right), \boldsymbol{e}_{k}\left(\xi_{k}\right)\right)$ for $k=0,1, \cdots$, $T-1$.

Besides, for any market mode and at any time period, we assume that (namely, Assumption 3) at least two assets among $N+1$ investment assets have different expected rate of returns. This is especially reasonable when there are a large number of assets in the market.

Assumption 3. $\mathrm{E}\left[\boldsymbol{P}_{k}(i)\right] \neq \mathbf{0}$ for $i \in \Pi$ and $k=0,1, \cdots, T-1$, where $\mathbf{0}$ is a $n$-dimension zero vector.

The multi-period mean-variance framework for DC pension investment management refers to the problem of finding the optimal admissible investment strategy such that the variance of the terminal wealth is minimized for a given expected terminal wealth level $d$. Specifically, we have the following formulation

$$
\left\{\begin{array}{l}
\min _{u \in \Theta_{0}}\left\{\operatorname{Var}\left[x_{T^{\tau}}\right]:=\mathrm{E}\left[x_{T^{\tau}}^{2}\right]-d^{2}\right\}, \\
\text { s.t. } \quad \mathrm{E}\left[x_{T^{\tau}}\right]=d, \\
\quad x_{k+1}=x_{k} e_{k}^{0}\left(\xi_{k}\right)+c y_{k} e_{k}^{0}\left(\xi_{k}\right)+P^{\prime}{ }_{k}\left(\xi_{k}\right) u_{k}, \\
\quad y_{k+1}=b_{k}\left(\xi_{k}\right) y_{k}, k=0,1, \cdots, T-1 .
\end{array}\right.
$$

The solution $\mathrm{u}^{*}=\left\{\boldsymbol{u}_{k}^{*} ; k=0,1, \cdots, T-1\right\}$ of Problem (7) with respect to $d \geq d_{\sigma_{\min }}$ is called an efficient investment strategy, where $d_{\sigma_{\min }}$ is the expected 
terminal wealth corresponding to the global minimum variance of terminal wealth over all admissible strategies. The point $\left(\operatorname{Var}\left[x_{T^{\tau}}\right], d\right)$ corresponding to an efficient investment strategy on the variance-mean space is called an efficient point. The set of all the efficient points forms the efficient frontier.

\section{Solution scheme}

Now we proceed with the solution of (7) which involves two steps. The first step is to remove the expected terminal wealth constraint by using the Lagrange method. The second step is to apply the dynamic programming.

For convenience, we define $p_{0}=0$. By the law of total probability and under Assumption 2, we have

$$
\left\{\begin{array}{l}
\mathrm{E}\left[x_{T^{\tau}}\right]=\sum_{s=0}^{T} \mathrm{E}\left[x_{T^{\tau}} \mid T^{\tau}=s\right] \operatorname{Pr}\left(T^{\tau}=s\right)=\mathrm{E}\left[\sum_{s=0}^{T} p_{s} x_{s}\right] \\
\mathrm{E}\left[x_{T^{\tau}}^{2}\right]=\sum_{s=0}^{T} \mathrm{E}\left[x_{T^{\tau}}^{2} \mid T^{\tau}=s\right] \operatorname{Pr}\left(T^{\tau}=s\right)=\mathrm{E}\left[\sum_{s=0}^{T} p_{s} x_{s}^{2}\right] .
\end{array}\right.
$$

Then Problem (7) is equivalent to

$$
\left\{\begin{aligned}
\min _{u \in \Theta_{0}} & \left\{\mathrm{E}\left[\sum_{s=0}^{T} p_{s} x_{s}^{2}\right]-d^{2}\right\} \\
\text { s.t. } & \mathrm{E}\left[\sum_{s=0}^{T} p_{s} x_{s}\right]=d \\
& x_{k+1}=x_{k} e_{k}^{0}\left(\xi_{k}\right)+c y_{k} e_{k}^{0}\left(\xi_{k}\right)+P^{\prime}{ }_{k}\left(\xi_{k}\right) u_{k} \\
& y_{k+1}=b_{k}\left(\xi_{k}\right) y_{k}, k=0,1, \cdots, T-1 .
\end{aligned}\right.
$$

Then equality constraint $\mathrm{E}\left[\sum_{s=0}^{T} p_{s} x_{s}\right]=d$ in Problem (9) can be eliminated by the Lagrange method. Introducing a Lagrange multiplier $2 \mu$, we consider the following optimization problem instead,

$$
\left\{\begin{array}{c}
\min _{u \in \Theta_{0}}\left\{\mathrm{E}\left[\sum_{s=0}^{T} p_{s} x_{s}^{2}\right]-d^{2}+2 \mu\left(\mathrm{E}\left[\sum_{s=0}^{T} p_{s} x_{s}\right]-d\right)\right\}, \\
\text { s.t. } \quad x_{k+1}=x_{k} e_{k}^{0}\left(\xi_{k}\right)+c y_{k} e_{k}^{0}\left(\xi_{k}\right)+P^{\prime}{ }_{k}\left(\xi_{k}\right) u_{k}, \\
y_{k+1}=b_{k}\left(\xi_{k}\right) y_{k}, k=0,1, \cdots, T-1 .
\end{array}\right.
$$

Taking

$$
\begin{gathered}
\mathrm{E}\left[\sum_{s=0}^{T} p_{s} x_{s}^{2}\right]-d^{2}+2 \mu\left(\mathrm{E}\left[\sum_{s=0}^{T} p_{s} x_{s}\right]-d\right) \\
=\mathrm{E}\left[\sum_{s=0}^{T}\left(p_{s} x_{s}^{2}+2 \mu p_{s} x_{s}\right)\right]-d^{2}-2 \mu d
\end{gathered}
$$


and since $\left(-d^{2}-2 \mu d\right)$ is fixed, Problem (10) is equivalent to the following optimization problem in the sense that both problems share the same optimal solution

$$
\left\{\begin{array}{c}
\min _{\Theta_{0}} \mathrm{E}\left[\sum_{s=0}^{T}\left(p_{s} x_{s}^{2}+2 \mu p_{s} x_{s}\right)\right] \\
\text { s.t. } \quad x_{k+1}=x_{k} e_{k}^{0}\left(\xi_{k}\right)+c y_{k} e_{k}^{0}\left(\xi_{k}\right)+P^{\prime}{ }_{k}\left(\xi_{k}\right) u_{k} \\
\quad y_{k+1}=b_{k}\left(\xi_{k}\right) y_{k}, k=0,1, \cdots, T-1 .
\end{array}\right.
$$

Hence, dynamic programming approach can be applied directly to solve Problem (12).

Let $f_{k}\left(x_{k}, y_{k}, \xi_{k}\right)$ be the optimal value function of Problem (12) starting from time $k$ with state variables $x_{k}, y_{k}$, and market state $\xi_{k}$, that is

$$
\left\{\begin{array}{c}
f_{k}\left(x_{k}, y_{k}, \xi_{k}\right)=\min _{u \in \Theta_{k}} \mathrm{E}\left[\sum_{s=k}^{T}\left(p_{s} x_{s}^{2}+2 \mu p_{s} x_{s}\right) \mid\left(x_{k}, y_{k}, \xi_{k}\right)\right] \\
\text { s.t. } \quad x_{s+1}=x_{s} e_{k}^{0}\left(\xi_{s}\right)+c y_{s} e_{s}^{0}\left(\xi_{s}\right)+P^{\prime}{ }_{s}\left(\xi_{s}\right) u_{s}, \\
y_{s+1}=b_{s}\left(\xi_{s}\right) y_{s}, s=k, k+1, \cdots, T-1 .
\end{array}\right.
$$

Similar to Costa and Araujo (2008) and Çanakoglu and Özekici (2010), according to dynamic programming principle, we have the Bellman equation for Problem (12) as follows

$$
\left\{\begin{aligned}
& f_{k}\left(x_{k}, y_{k}, \xi_{k}\right) \\
= & \min _{\boldsymbol{u}_{k}} \mathrm{E}\left[p_{k} x_{k}^{2}+2 \mu p_{k} x_{k}+f_{k+1}\left(x_{k+1}, y_{k+1}, \xi_{k+1}\right) \mid\left(x_{k}, y_{k}, \xi_{k}\right)\right] \\
= & p_{k} x_{k}^{2}+2 p_{k} \mu x_{k} \\
& +\min _{u_{k}} \sum_{j=1}^{m} \mathrm{E}\left[f_{k+1}\left(x_{k} e_{k}^{0}\left(\xi_{k}\right)+c y_{k} e_{k}^{0}\left(\xi_{k}\right)+\boldsymbol{P}_{k}^{\prime}\left(\xi_{k}\right) \boldsymbol{u}_{k}, b_{k}\left(\xi_{k}\right) y_{k}, j\right)\right] q_{\xi_{k}, j}(k), \\
& f_{T}\left(x_{T}, y_{T}, \xi_{T}\right)=p_{T} x_{T}^{2}+2 p_{T} \mu x_{T} .
\end{aligned}\right.
$$

Obviously, set $k=0$, then $f_{0}\left(x_{0}, y_{0}, \xi_{0}\right)$ and $\left(f_{0}\left(x_{0}, y_{0}, \xi_{0}\right)-d^{2}-2 \mu d\right)$ are the optimal values of Problem (12) and (10), respectively.

For later use, for any $i \in \Pi$ and $k=0,1, \cdots, T-1$, let

$$
\left\{\begin{array}{l}
A_{k}(i)=\mathrm{E}\left[\left(e_{k}^{0}(i)\right)^{2}\right]-\mathrm{E}\left[e_{k}^{0}(i) \boldsymbol{P}_{k}^{\prime}(i)\right] \mathrm{E}^{-1}\left[\boldsymbol{P}_{k}(i) \boldsymbol{P}_{k}^{\prime}(i)\right] \mathrm{E}\left[e_{k}^{0}(i) \boldsymbol{P}_{k}(i)\right], \\
B_{k}(i)=\mathrm{E}\left[b_{k}^{2}(i)\right]-\mathrm{E}\left[b_{k}(i) \boldsymbol{P}_{k}^{\prime}(i)\right] \mathrm{E}^{-1}\left[\boldsymbol{P}_{k}(i) \boldsymbol{P}_{k}^{\prime}(i)\right] \mathrm{E}\left[b_{k}(i) \boldsymbol{P}_{k}(i)\right] \\
C_{k}(i)=\mathrm{E}\left[e_{k}^{0}(i) b_{k}(i)\right]-\mathrm{E}\left[e_{k}^{0}(i) \boldsymbol{P}_{k}^{\prime}(i)\right] \mathrm{E}^{-1}\left[\boldsymbol{P}_{k}(i) \boldsymbol{P}_{k}^{\prime}(i)\right] \mathrm{E}\left[b_{k}(i) \boldsymbol{P}_{k}(i)\right], \\
D_{k}(i)=\mathrm{E}\left[\boldsymbol{P}_{k}^{\prime}(i)\right] \mathrm{E}^{-1}\left[\boldsymbol{P}_{k}(i) \boldsymbol{P}_{k}^{\prime}(i)\right] \mathrm{E}\left[\boldsymbol{P}_{k}(i)\right] \\
J_{k}(i)=\mathrm{E}\left[e_{k}^{0}(i)\right]-\left[e_{k}^{0}(i) \boldsymbol{P}_{k}^{\prime}(i)\right] \mathrm{E}^{-1}\left[\boldsymbol{P}_{k}(i) \boldsymbol{P}_{k}^{\prime}(i)\right] \mathrm{E}\left[\boldsymbol{P}_{k}(i)\right] \\
M_{k}(i)=\mathrm{E}\left[b_{k}(i)\right]-\mathrm{E}\left[b_{k}(i) \boldsymbol{P}_{k}^{\prime}(i)\right] \mathrm{E}^{-1}\left[\boldsymbol{P}_{k}(i) \boldsymbol{P}_{k}^{\prime}(i)\right] \mathrm{E}\left[\boldsymbol{P}_{k}(i)\right] .
\end{array}\right.
$$

In order to obtain the explicit expression of $f_{k}\left(x_{k}, y_{k}, \xi_{k}\right)$ for any $i \in \Pi$ and $k=$ $0,1, \cdots, T$, we construct series of $w_{k}(i), h_{k}(i), \alpha_{k}(i), \phi_{k}(i), \gamma_{k}(i)$, and $g_{k}(i)$ satisfying 
the following recursive relations and boundary conditions

$$
\begin{aligned}
& w_{k}(i)=p_{k}+\overline{w_{k+1}(i)} A_{k}(i), w_{T}(i)=p_{T}, \\
& h_{k}(i)=p_{k}+\overline{h_{k+1}(i)} J_{k}(i), h_{T}(i)=p_{T}, \\
& \alpha_{k}(i)=\overline{\alpha_{k+1}(i)}-\frac{\left(\overline{h_{k+1}(i)}\right)^{2}}{\overline{w_{k+1}(i)}} D_{k}(i), \alpha_{T}(i)=0 \text {, } \\
& \phi_{k}(i)=\overline{\phi_{k+1}(i)} C_{k}(i)+\overline{w_{k+1}(i)} A_{k}(i), \phi_{T}(i)=0, \\
& \left\{\begin{array}{l}
\gamma_{k}(i)=\overline{\gamma_{k+1}(i)} \mathrm{E}\left[b_{k}^{2}(i)\right]+\overline{w_{k+1}(i)} A_{k}(i)-\frac{\left(\overline{\phi_{k+1}(i)}\right)^{2}}{\overline{w_{k+1}(i)}} B_{k}(i)+2 \overline{\phi_{k+1}(i)} C_{k}(i), \\
\gamma_{T}(i)=0,
\end{array}\right. \\
& \left\{\begin{array}{l}
g_{k}(i)=\overline{g_{k+1}(i)} \mathrm{E}\left[b_{k}(i)\right]+\overline{h_{k+1}(i)} J_{k}(i)-\frac{\overline{\frac{\phi_{k+1}(i)}{h_{k+1}(i)}}}{\overline{w_{k+1}(i)}} M_{k}(i), \\
g_{T}(i)=0
\end{array}\right.
\end{aligned}
$$

where

$$
\begin{cases}\overline{w_{k+1}(i)} & =\sum_{j=1}^{m} w_{k+1}(j) q_{i j}(k), \overline{h_{k+1}(i)}=\sum_{j=1}^{m} h_{k+1}(j) q_{i j}(k), \\ \overline{\phi_{k+1}(i)} & =\sum_{j=1}^{m} \phi_{k+1}(j) q_{i j}(k), \overline{\alpha_{k+1}(i)}=\sum_{j=1}^{m} \alpha_{k+1}(j) q_{i j}(k), \\ \overline{\gamma_{k+1}(i)} & =\sum_{j=1}^{m} \gamma_{k+1}(j) q_{i j}(k), \overline{g_{k+1}(i)}=\sum_{j=1}^{m} g_{k+1}(j) q_{i j}(k) .\end{cases}
$$

Before we attempt the solution for (14), we present the following result on the property of $w_{k}(i)$.

Proposition 1. For any $i \in \Pi$ and $k=0,1, \cdots, T$, we have $w_{k}(i)>0$.

Proof. This can be proved by the mathematical induction. For $k=T$, according to (16) and (6), we have $w_{T}(i)=p_{T}=e^{-\int_{0}^{T-1} \lambda(s) d s}>0$ for all $i \in \Pi$.

Now, we prove $w_{k}(i)>0$ for any $i \in \Pi$ given the assumption that $w_{k+1}(i)>0$ for any $i \in \Pi$. Note that $q_{i j}(k) \geq 0$ for $i, j \in \Pi$ and $\sum_{j=1}^{S} q_{i j}(k)=1$ for $i \in \Pi$, then we have $\overline{w_{k+1}(i)}=\sum_{j=1}^{S} w_{k+1}(j) q_{i j}(k)>0$ for $i \in \Pi$. It is known from Li and $\mathrm{Ng}(2000)$ and Chen and Yang (2011) that under Assumption 1

$$
A_{k}(i)=\mathrm{E}\left[\left(e_{k}^{0}(i)\right)^{2}\right]-\mathrm{E}\left[e_{k}^{0}(i) \boldsymbol{P}_{k}^{\prime}(i)\right] \mathrm{E}^{-1}\left[\boldsymbol{P}_{k}(i) \boldsymbol{P}_{k}^{\prime}(i)\right] \mathrm{E}\left[e_{k}^{0}(i) \boldsymbol{P}_{k}(i)\right]>0
$$

for $i \in \Pi$. By $p_{k} \geq 0$ and equation (16), for all $i \in \Pi$ we have

$$
w_{k}(i)=p_{k}+\overline{w_{k+1}(i)} A_{k}(i)>0 .
$$

By principle of mathematical induction, the proposition is proved. 
Based on Proposition 1, we investigate the optimal value function in the following theorem.

Theorem 1. For simplicity, let $x=x_{k}, y=y_{k}$ and $i=\xi_{k}$. Then for $k=0,1, \cdots, T$ and $i \in \Pi$, the solution to Bellman equation (14), namely the optimal value function of Problem (12) is given by

$$
\begin{aligned}
f_{k}(x, y, i)= & w_{k}(i) x^{2}+2 \phi_{k}(i) x c y+\gamma_{k}(i) c^{2} y^{2} \\
& +2 h_{k}(i) \mu x+2 g_{k}(i) \mu c y+\alpha_{k}(i) \mu^{2} .
\end{aligned}
$$

Moreover, the optimal strategy is

$$
\begin{aligned}
& \boldsymbol{u}_{k}=-\mathrm{E}^{-1}\left[\boldsymbol{P}_{k}(i) \boldsymbol{P}_{k}^{\prime}(i)\right]\left((x+c y) \mathrm{E}\left[e_{k}^{0}(i) \boldsymbol{P}_{k}^{\prime}(i)\right]\right.
\end{aligned}
$$

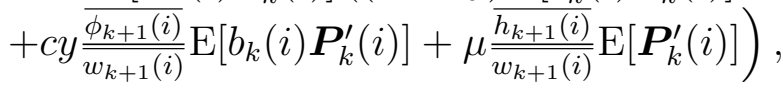

where $w_{k}(i), h_{k}(i), \alpha_{k}(i), \phi_{k}(i), \gamma_{k}(i)$, and $g_{k}(i)$ are defined by (16)-(21).

Proof. We first prove that optimal value function of Problem (12) is given by (23), where the backward mathematical induction on $k$ is applied.

For $k=T$, by the boundary conditions of (16)-(21), we have

$$
\begin{array}{r}
w_{T}(i) x^{2}+2 \phi_{T}(i) c x y+\gamma_{T}(i) c^{2} y^{2}+2 h_{T}(i) \mu x \\
+2 g_{T}(i) \mu c y+\alpha_{T}(i) \mu^{2}=p_{T} x^{2}+2 p_{T} \mu x .
\end{array}
$$

On the other hand, it is known from the boundary condition of Bellman equation (14) that $f_{T}(x, y, i)=p_{T} x^{2}+2 p_{T} \mu x$. Therefore, (23) holds for $k=T$.

Suppose that (23) holds for $k+1$, that is

$$
\begin{aligned}
f_{k+1}(x, y, i)= & w_{k+1}(i) x^{2}+\gamma_{k+1}(i) c^{2} y^{2}+2 \phi_{k+1}(i) c x y \\
& +2 h_{k+1}(i) \mu x+2 g_{k+1}(i) \mu c y+\alpha_{k+1}(i) \mu^{2} .
\end{aligned}
$$

Then for $k$, by Bellman equation (14) and taking notice of (22), it follows that

$$
\begin{aligned}
& f_{k}(x, y, i)=p_{k} x^{2}+2 p_{k} \mu x \\
& +\min _{\boldsymbol{u}_{k}} \sum_{j=1}^{m} \mathrm{E}\left[f_{k+1}\left(x e_{k}^{0}(i)+c y e_{k}^{0}(i)+\boldsymbol{P}_{k}^{\prime}(i) \boldsymbol{u}_{k}, b_{k}(i) y_{k}, j\right)\right] q_{i j}(k) \\
& =p_{k} x^{2}+2 p_{k} \mu x+\min _{\boldsymbol{u}_{k}} \mathrm{E} \overline{w_{k+1}(i)}\left(x e_{k}^{0}(i)+c y e_{k}^{0}(i)+\boldsymbol{P}_{k}^{\prime}(i) \boldsymbol{u}_{k}\right)^{2} \\
& +\overline{\gamma_{k+1}(i)} c^{2} b_{k}^{2}(i) y^{2}+2 \overline{\phi_{k+1}(i)} c\left(x e_{k}^{0}(i)+c y e_{k}^{0}(i)+\boldsymbol{P}_{k}^{\prime}(i) \boldsymbol{u}_{k}\right) b_{k}(i) y \\
& \left.+2 c \mu \overline{g_{k+1}(i)} b_{k}(i) y+2 \overline{h_{k+1}(i)} \mu\left(x e_{k}^{0}(i)+c y e_{k}^{0}(i)+\boldsymbol{P}_{k}^{\prime}(i) \boldsymbol{u}_{k}\right)+\overline{\alpha_{k+1}(i)} \mu^{2}\right] \\
& =p_{k} x^{2}+2 p_{k} \mu x+\overline{w_{k+1}(i)} x^{2} \mathrm{E}\left[\left(e_{k}^{0}(i)\right)^{2}\right]+\overline{w_{k+1}(i)} c^{2} y^{2} \mathrm{E}\left[\left(e_{k}^{0}(i)\right)^{2}\right] \\
& +2 \overline{w_{k+1}(i)} c y x \mathrm{E}\left[\left(e_{k}^{0}(i)\right)^{2}\right]+\overline{\gamma_{k+1}(i)} c^{2} y^{2} \mathrm{E}\left[b_{k}^{2}(i)\right]+\overline{\alpha_{k+1}(i)} \mu^{2} \\
& +2 \overline{\phi_{k+1}(i)} c y x \mathrm{E}\left[b_{k}(i) e_{k}^{0}(i)\right]+2 \overline{\phi_{k+1}(i)} c^{2} y^{2} \mathrm{E}\left[b_{k}(i) e_{k}^{0}(i)\right] \\
& +2 \overline{g_{k+1}(i)} \mu \mathrm{E}\left[b_{k}(i)\right] c y+2 \overline{h_{k+1}(i)} \mu x \mathrm{E}\left[e_{k}^{0}(i)\right]+2 \overline{h_{k+1}(i)} \mu c y \mathrm{E}\left[e_{k}^{0}(i)\right] \\
& +\min _{\boldsymbol{u}_{k}}\left\{\overline{w_{k+1}(i)} \boldsymbol{u}_{k}^{\prime} \mathrm{E}\left[\boldsymbol{P}_{k}(i) \boldsymbol{P}_{k}^{\prime}(i)\right] \boldsymbol{u}_{k}+2\left(\overline{w_{k+1}(i)}(x+c y)\right.\right. \\
& \left.\left.\quad \times \mathrm{E}\left[e_{k}^{0}(i) \boldsymbol{P}_{k}^{\prime}(i)\right]+\overline{\phi_{k+1}(i)} c y \mathrm{E}\left[b_{k}(i) \boldsymbol{P}_{k}^{\prime}(i)\right]+\overline{h_{k+1}(i)} \mu \mathrm{E}\left[\boldsymbol{P}_{k}^{\prime}(i)\right]\right) \boldsymbol{u}_{k}\right\} .
\end{aligned}
$$


It is known from Proposition 1 that $w_{k+1}(i)>0$ for all $i \in \Pi$. On the other hand, by $\mathrm{Li}$ and $\mathrm{Ng}(2000)$ it follows that $\mathrm{E}\left[\boldsymbol{P}_{k}(i) \boldsymbol{P}_{k}^{\prime}(i)\right]$ is positive definite given Assumption 1. Therefore, the first order condition (also is sufficient condition) on $\boldsymbol{u}_{k}$ in (25) gives the optimal strategy

$$
\begin{aligned}
\boldsymbol{u}_{k}= & -\mathrm{E}^{-1}\left[\boldsymbol{P}_{k}(i) \boldsymbol{P}_{k}^{\prime}(i)\right]\left((x+c y) \mathrm{E}\left[e_{k}^{0}(i) \boldsymbol{P}_{k}^{\prime}(i)\right]\right. \\
& \left.+c y \frac{\overline{\phi_{k+1}(i)}}{\overline{w_{k+1}(i)}} \mathrm{E}\left[b_{k}(i) \boldsymbol{P}_{k}^{\prime}(i)\right]+\mu \frac{\overline{h_{k+1}(i)}}{w_{k+1}(i)} \mathrm{E}\left[\boldsymbol{P}_{k}^{\prime}(i)\right]\right) .
\end{aligned}
$$

Plugging (26) into (25), we obtain

$$
\begin{aligned}
& f_{k}(x, y, i)=p_{k} x^{2}+2 p_{k} \mu x+\overline{w_{k+1}(i)} x^{2} \mathrm{E}\left[\left(e_{k}^{0}(i)\right)^{2}\right] \\
& +\overline{w_{k+1}(i)} c^{2} y^{2} \mathrm{E}\left[\left(e_{k}^{0}(i)\right)^{2}\right]+2 \overline{w_{k+1}(i)} c y x \mathrm{E}\left[\left(e_{k}^{0}(i)\right)^{2}\right] \\
& +\overline{\gamma_{k+1}(i)} c^{2} y^{2} \mathrm{E}\left[b_{k}^{2}(i)\right]+\overline{\alpha_{k+1}(i)} \mu^{2}+2 \overline{\phi_{k+1}(i)} c y x \mathrm{E}\left[b_{k}(i) e_{k}^{0}(i)\right] \\
& +2 \overline{\phi_{k+1}(i)} c^{2} y^{2} \mathrm{E}\left[b_{k}(i) e_{k}^{0}(i)\right]+2 \overline{g_{k+1}(i)} \mu \mathrm{E}\left[b_{k}(i)\right] c y \\
& +2 \overline{h_{k+1}(i)} \mu c y \mathrm{E}\left[e_{k}^{0}(i)\right]+2 \overline{h_{k+1}(i)} \mu x \mathrm{E}\left[e_{k}^{0}(i)\right]-\left((x+c y) \overline{w_{k+1}(i)}\right. \\
& \left.\times \mathrm{E}\left[e_{k}^{0}(i) \boldsymbol{P}^{\prime}(i)_{k}\right]+c y \overline{\phi_{k+1}(i)} \mathrm{E}\left[b_{k}(i) \boldsymbol{P}_{k}^{\prime}(i)\right]+\mu \overline{h_{k+1}(i)} \mathrm{E}\left[\boldsymbol{P}_{k}^{\prime}(i)\right]\right) \\
& \times \mathrm{E}^{-1}\left[\boldsymbol{P}_{k}(i) \boldsymbol{P}_{k}^{\prime}(i)\right]\left((x+c y) \mathrm{E}\left[e_{k}^{0}(i) \boldsymbol{P}_{k}^{\prime}(i)\right]\right. \\
& \left.+c y \frac{\overline{\frac{\phi_{k+1}(i)}{w_{k+1}(i)}}}{\mathrm{E}}\left[b_{k}(i) \boldsymbol{P}_{k}^{\prime}(i)\right]+\mu \frac{\overline{h_{k+1}(i)}}{w_{k+1}(i)} \mathrm{E}\left[\boldsymbol{P}_{k}^{\prime}(i)\right]\right) \text {. }
\end{aligned}
$$

Simplifying the above formula and taking notice of (15), it follows that

$$
\begin{aligned}
& f_{k}(x, y, i)=\left(p_{k}+\overline{w_{k+1}(i)} A_{k}(i)\right) x^{2}+2\left(p_{k}+\overline{h_{k+1}(i)} J_{k}(i)\right) \mu x \\
& \quad+2 x c y\left(\overline{w_{k+1}(i)} A_{k}(i)+\overline{\phi_{k+1}(i)} C_{k}(i)\right)+\mu^{2}\left(\overline{\alpha_{k+1}(i)}-\frac{\left(\overline{h_{k+1}(i)}\right)^{2}}{\overline{w_{k+1}(i)}} D_{k}(i)\right) \\
& \quad+c^{2} y^{2}\left(\overline{w_{k+1}(i)} A_{k}(i)+\overline{\gamma_{k+1}(i)} \mathrm{E}\left[b_{k}^{2}(i)\right]-\frac{\left(\overline{\phi_{k+1}(i)}\right)^{2}}{\overline{w_{k+1}(i)}} B_{k}(i)+2 \overline{\phi_{k+1}(i)} C_{k}(i)\right) \\
& \quad+2 \mu c y\left(\overline{h_{k+1}(i)} J_{k}(i)+\overline{g_{k+1}(i)} \mathrm{E}\left[b_{k}(i)\right]-\frac{\left.\overline{\phi_{k+1}(i)} \frac{\overline{h_{k+1}(i)}}{\overline{w_{k+1}(i)}} M_{k}(i)\right) .}{}\right.
\end{aligned}
$$

Therefore, according to (16)-(21), we have

$$
\begin{aligned}
f_{k}(x, y, i)= & w_{k}(i) x^{2}+\gamma_{k}(i) c^{2} y^{2}+2 \phi_{k}(i) c x y \\
& +2 h_{k}(i) \mu x+2 g_{k}(i) \mu c y+\alpha_{k}(i) \mu^{2} .
\end{aligned}
$$

This means that (23) holds for $k$.

By the Principle of Mathematical Induction, (23) holds for all $k=0,1, \cdots, T$.

According to the proof above (see formula (26)), we also have proved the fact that the optimal strategy for Problem (12) is given by (24).

In the following, we study the computational formula for series of $w_{k}(i), h_{k}(i)$, $\alpha_{k}(i), \phi_{k}(i), \gamma_{k}(i)$, and $g_{k}(i)$. Firstly we derive the expressions for $w_{k}(i)$ and $h_{k}(i)$. To this end, let

$$
\left\{\begin{array}{l}
\boldsymbol{w}_{\boldsymbol{k}}=\left(w_{k}(1), w_{k}(2), \cdots, w_{k}(m)\right)^{\prime}, \boldsymbol{h}_{k}=\left(h_{k}(1), h_{k}(2), \cdots, h_{k}(m)\right)^{\prime}, \\
\boldsymbol{A}_{k}=\left(A_{k}(1), A_{k}(2), \cdots, A_{k}(m)\right), \boldsymbol{J}_{k}=\left(J_{k}(1), J_{k}(2), \cdots, J_{k}(m)\right) .
\end{array}\right.
$$


Then, (16) and (17) can be reformulated as

$$
\begin{gathered}
\boldsymbol{w}_{k}=\operatorname{diag}\left(\boldsymbol{A}_{k}\right) \mathbb{Q}(k) \boldsymbol{w}_{k+1}+p_{k} \mathbf{1}, \boldsymbol{w}_{T}=p_{T} \mathbf{1}, \\
\boldsymbol{h}_{k}=\operatorname{diag}\left(\boldsymbol{J}_{k}\right) \mathbb{Q}(k) \boldsymbol{h}_{k+1}+p_{k} \mathbf{1}, \boldsymbol{h}_{T}=p_{T} \mathbf{1},
\end{gathered}
$$

where $\mathbf{1}=(1,1, \cdots, 1)^{\prime} \in \mathrm{R}^{m}$ and $\operatorname{diag}\left(l_{1}, l_{2}, \cdots, l_{m}\right)$ denote a diagonal matrix of order $m \times m$ with the diagonal elements $l_{1}, l_{2}, \cdots, l_{m}$.

For convenience, throughout this paper, we define $\prod_{j=k}^{k-1}(\cdot)=1$ and $\sum_{i=k}^{k-1}(\cdot)=0$. The following proposition gives the expression of $\boldsymbol{w}_{k}$ and $\boldsymbol{h}_{k}$ for $k=0,1, \cdots, T$.

Proposition 2. For $k=0,1, \cdots, T$, we have

$$
\boldsymbol{w}_{k}=\sum_{s=k}^{T} p_{s}\left(\prod_{t=k}^{s-1} \operatorname{diag}\left(\boldsymbol{A}_{t}\right) \mathbb{Q}(t)\right) \mathbf{1}, \boldsymbol{h}_{k}=\sum_{s=k}^{T} p_{s}\left(\prod_{t=k}^{s-1} \operatorname{diag}\left(\boldsymbol{J}_{t}\right) \mathbb{Q}(t)\right) \mathbf{1}
$$

PROOF. we only need to prove the first formula, the other formula can be proved in a similar way. The Mathematical induction method is used to proved this proposition. For $k=T$, it is easy to verify

$$
\sum_{s=T}^{T} p_{s}\left(\prod_{t=T}^{s-1} \operatorname{diag}\left(\boldsymbol{A}_{t}\right) \mathbb{Q}(t)\right) \mathbf{1}=p_{T}\left(\prod_{t=T}^{T-1} \operatorname{diag}\left(\boldsymbol{A}_{t}\right) \mathbb{Q}(t)\right) \mathbf{1}=p_{T} \mathbf{1}=\boldsymbol{w}_{T}
$$

Now suppose that $\boldsymbol{w}_{k+1}=\sum_{s=k+1}^{T} p_{s}\left(\prod_{t=k+1}^{s-1} \operatorname{diag}\left(\boldsymbol{A}_{t}\right) \mathbb{Q}(t)\right) \mathbf{1}$. According to (28), we have

$$
\begin{aligned}
\boldsymbol{w}_{k} & =\operatorname{diag}\left(\boldsymbol{A}_{k}\right) \mathbb{Q}(k) \boldsymbol{w}_{k+1}+p_{k} \mathbf{1} \\
& =\operatorname{diag}\left(\boldsymbol{A}_{k}\right) \mathbb{Q}(k) \sum_{s=k+1}^{T} p_{s}\left(\prod_{t=k+1}^{s-1} \operatorname{diag}\left(\boldsymbol{A}_{t}\right) \mathbb{Q}(t)\right) \mathbf{1}+p_{k} \mathbf{1} \\
& =\sum_{s=k+1}^{T} p_{s}\left(\prod_{t=k}^{s-1} \operatorname{diag}\left(\boldsymbol{A}_{t}\right) \mathbb{Q}(t)\right) \mathbf{1}+p_{k}\left(\prod_{t=k}^{k-1} \operatorname{diag}\left(\boldsymbol{A}_{t}\right) \mathbb{Q}(t)\right) \mathbf{1} \\
& =\sum_{s=k}^{T} p_{s}\left(\prod_{t=k}^{s-1} \operatorname{diag}\left(\boldsymbol{A}_{t}\right) \mathbb{Q}(t)\right) \mathbf{1} .
\end{aligned}
$$

Therefore, according to the mathematical induction, $\boldsymbol{w}_{k}=\sum_{s=k}^{T} p_{s}\left(\prod_{t=k}^{s-1} \operatorname{diag}\left(\boldsymbol{A}_{t}\right) \mathbb{Q}(t)\right) \mathbf{1}$, for all $k=0,1, \cdots, T$. The proposition is proved.

After obtaining the expression of $\boldsymbol{w}_{k}$ and $\boldsymbol{h}_{k}$, the $i$ th $(i \in \Pi)$ component of $\boldsymbol{w}_{k}$ and $\boldsymbol{h}_{k}$ are $w_{k}(i)$ and $h_{k}(i)$, respectively. In the following, we derive the expressions for series of $\phi_{k}(i)$ and $\alpha_{k}(i)$ for $i \in \Pi$. Let

$$
\left\{\begin{array}{l}
\boldsymbol{\alpha}_{k}=\left(\alpha_{k}(1), \alpha_{k}(2), \cdots, \alpha_{k}(m)\right)^{\prime}, \boldsymbol{\phi}_{k}=\left(\phi_{k}(1), \phi_{k}(2), \cdots, \phi_{k}(m)\right)^{\prime}, \\
\Psi_{k}(i)=-\frac{\left(\overline{h_{k+1}(i)}\right)^{2}}{\overline{w_{k+1}(i)}} D_{k}(i), i \in \Pi, \boldsymbol{\Psi}_{k}=\left(\Psi_{k}(1), \Psi_{k}(2), \cdots, \Psi_{k}(m)\right)^{\prime} \\
\boldsymbol{C}_{k}=\left(C_{k}(1), C_{k}(2), \cdots, C_{k}(m)\right), \boldsymbol{\Theta}_{k}=\operatorname{diag}\left(\boldsymbol{A}_{k}\right) \mathbb{Q}(k) \boldsymbol{w}_{k+1} .
\end{array}\right.
$$


Then formulations (18) and (19) can be reformulated as

$$
\begin{gathered}
\boldsymbol{\alpha}_{k}=\mathbb{Q}(k) \boldsymbol{\alpha}_{k+1}+\boldsymbol{\Psi}_{k}, \boldsymbol{\alpha}_{T}=\mathbf{0} \\
\boldsymbol{\phi}_{k}=\operatorname{diag}\left(\boldsymbol{C}_{k}\right) \mathbb{Q}(k) \boldsymbol{\phi}_{k+1}+\boldsymbol{\Theta}_{k}, \boldsymbol{\phi}_{T}=\mathbf{0}
\end{gathered}
$$

The following proposition gives the expressions of vector series of $\boldsymbol{\alpha}_{k}$ and $\boldsymbol{\phi}_{k}$, namely for series of $\phi_{k}(i)$ and $\alpha_{k}(i)$ for $i \in \Pi$ and $k=0,1, \cdots, T$.

Proposition 3. for $k=0,1, \cdots, T$, we have

$$
\boldsymbol{\alpha}_{k}=\sum_{s=k}^{T-1}\left(\prod_{t=k}^{s-1} \mathbb{Q}(t)\right) \boldsymbol{\Psi}_{s}, \boldsymbol{\phi}_{k}=\sum_{s=k}^{T-1}\left(\prod_{t=k}^{s-1} \operatorname{diag}\left(\boldsymbol{C}_{t}\right) \mathbb{Q}(t)\right) \boldsymbol{\Theta}_{s} .
$$

The proof of Proposition 3 is similar to that of Proposition 2, therefore we omit it.

After obtaining the expressions for $w_{k}(i), h_{k}(i), \phi_{k}(i)$ and $\alpha_{k}(i)$, finally, we derive the expressions for $\gamma_{k}(i)$ and $g_{k}(i)$. Let

$$
\left\{\begin{array}{l}
\gamma_{k}=\left(\gamma_{k}(1), \gamma_{k}(2), \cdots, \gamma_{k}(m)\right)^{\prime}, \boldsymbol{g}_{k}=\left(g_{k}(1), g_{k}(2), \cdots, g_{k}(m)\right)^{\prime}, \\
\boldsymbol{\Lambda}_{k}=\mathrm{E}\left[\left(b_{k}^{2}(1), b_{k}^{2}(2), \cdots, b_{k}^{2}(m)\right)\right], \boldsymbol{\Delta}_{k}=\mathrm{E}\left[\left(b_{k}(1), b_{k}(2), \cdots, b_{k}(m)\right)\right], \\
F_{k}(i)=\overline{w_{k+1}(i)} A_{k}(i)-\frac{\left(\overline{\phi_{k+1}(i)}\right)^{2}}{w_{k+1}(i)} B_{k}(i)+2 \overline{\phi_{k+1}(i)} C_{k}(i), i \in \Pi, \\
N_{k}(i)=\overline{h_{k+1}(i)} J_{k}(i)-\frac{\overline{\phi_{k+1}(i) h_{k+1}(i)}}{\overline{w_{k+1}(i)}} M_{k}(i), i \in \Pi, \\
\boldsymbol{F}_{k}=\left(F_{k}(1), F_{k}(2), \cdots, F_{k}(m)\right)^{\prime}, \boldsymbol{N}_{k}=\left(N_{k}(1), N_{k}(2), \cdots, N_{k}(m)\right)^{\prime} .
\end{array}\right.
$$

Then formulation (20) and (21) can be reformulated as

$$
\begin{aligned}
& \boldsymbol{\gamma}_{k}=\operatorname{diag}\left(\boldsymbol{\Lambda}_{k}\right) \mathbb{Q}(k) \boldsymbol{\gamma}_{k+1}+\boldsymbol{F}_{k}, \boldsymbol{\gamma}_{T}=\mathbf{0}, \\
& \boldsymbol{g}_{k}=\operatorname{diag}\left(\boldsymbol{\Delta}_{k}\right) \mathbb{Q}(k) \boldsymbol{g}_{k+1}+\boldsymbol{N}_{k}, \boldsymbol{g}_{T}=\mathbf{0} .
\end{aligned}
$$

The following proposition gives the expression of vector series $\gamma_{k}$ and $\boldsymbol{g}_{k}$, namely for $\gamma_{k}(i)$ and $g_{k}(i)$ for $i \in \Pi$ and $k=0,1, \cdots, T$.

Proposition 4. For $k=0,1, \cdots, T$, we have

$$
\boldsymbol{\gamma}_{k}=\sum_{s=k}^{T-1}\left(\prod_{t=k}^{s-1} \operatorname{diag}\left(\boldsymbol{\Lambda}_{t}\right) \mathbb{Q}(t)\right) \boldsymbol{F}_{s}, \boldsymbol{g}_{k}=\sum_{s=k}^{T-1}\left(\prod_{t=k}^{s-1} \operatorname{diag}\left(\boldsymbol{\Delta}_{t}\right) \mathbb{Q}(t)\right) \boldsymbol{N}_{s}
$$




\section{Efficient investment strategy and efficient frontier}

It is known from the previous analysis in Section 3 that the optimal value of Problem (10) is

$$
H\left(x_{0}, y_{0}, \xi_{0}, \mu\right)=f_{0}\left(x_{0}, y_{0}, \xi_{0}\right)-d^{2}-2 \mu d .
$$

It follows from Theorem 1 that we have

$$
\begin{aligned}
& H\left(x_{0}, y_{0}, \xi_{0}, \mu\right) \\
= & w_{0}\left(\xi_{0}\right) x_{0}^{2}+2 \phi_{0}\left(\xi_{0}\right) c x_{0} y_{0}+\gamma_{0}\left(\xi_{0}\right) c^{2} y_{0}^{2}+2 h_{0}\left(\xi_{0}\right) \mu x_{0} \\
& +2 g_{0}\left(\xi_{0}\right) c \mu y_{0}+\alpha_{0}\left(\xi_{0}\right) \mu^{2}-d^{2}-2 \mu d \\
= & \alpha_{0}\left(\xi_{0}\right) \mu^{2}+2 \mu\left(h_{0}\left(\xi_{0}\right) x_{0}+g_{0}\left(\xi_{0}\right) c y_{0}-d\right)+w_{0}\left(\xi_{0}\right) x_{0}^{2} \\
& +2 \phi_{0}\left(\xi_{0}\right) c x_{0} y_{0}+\gamma_{0}\left(\xi_{0}\right) c^{2} y_{0}^{2}-d^{2} .
\end{aligned}
$$

According to the Lagrange dual theory (see Luenberger (1968)), the optimal value of Problem (7) (which is equivalent to Problem (9)) can be obtained by maximizing $H\left(x_{0}, y_{0}, \xi_{0}, \mu\right)$ over $\mu$, i.e.,

$$
\operatorname{Var}^{*}\left[x_{T^{\tau}}\right]=\max _{\mu} H\left(x_{0}, y_{0}, \xi_{0}, \mu\right) .
$$

In order to obtain the existence of the optimal solution to Problem (41), we give the following proposition.

Proposition 5. For any $i \in \Pi$ and $k=0,1, \cdots, T-1$, we have $\alpha_{k}(i)<0$.

Proof. This proposition is also proved by the backward mathematical induction. For $k=T-1$, According to (16)-(18), for any $i \in \Pi$ we have

$$
\alpha_{T-1}(i)=-\frac{\left(\overline{h_{T}(i)}\right)^{2}}{\overline{w_{T}(i)}} D_{T-1}(i)=-p_{T} D_{T-1}(i) .
$$

According to Li and Ng (2000) and Chen and Yang (2011), E[ $\left.\boldsymbol{P}_{T-1}(i) \boldsymbol{P}_{T-1}^{\prime}(i)\right]$ is positive definite under Assumption 1, then so is $\mathrm{E}^{-1}\left[\boldsymbol{P}_{T-1}(i) \boldsymbol{P}_{T-1}^{\prime}(i)\right]$. By Assumption 3 , we get $\mathrm{E}\left[\boldsymbol{P}_{T-1}(i)\right] \neq \mathbf{0}$, and hence

$$
D_{T-1}(i)=\mathrm{E}\left[\boldsymbol{P}_{T-1}^{\prime}(i)\right] \mathrm{E}^{-1}\left[\boldsymbol{P}_{T-1}(i) \boldsymbol{P}_{T-1}^{\prime}(i)\right] \mathrm{E}\left[\boldsymbol{P}_{T-1}(i)\right]>0,
$$

which along with $p_{T}>0$ give $\alpha_{T-1}(i)=-D_{T-1}(i) p_{T}<0$. This proves the proposition.

Now we prove $\alpha_{k}(i)<0$ for any $i \in \Pi$ under the assumption that $\alpha_{k+1}(i)<0$ for any $i \in \Pi$. By proposition $1, w_{k+1}(j)>0$ for any $j \in \Pi$. Note that $q_{i j}(k) \geq 0$ and $\sum_{j=1}^{S} q_{i j}(k)=1$ for $i, j \in \Pi$, we have $\overline{w_{k+1}(i)}=\sum_{j=1}^{S} w_{k+1}(j) q_{i j}(k)>0$ for all $i \in \Pi$. 
Similar to the previous analysis, $D_{k}(i)>0$. Obviously, $\left(\overline{h_{k+1}(i)}\right)^{2} \geq 0$. Therefore, according to (18), for all $i \in \Pi$ we have

$$
\alpha_{k}(i)=\overline{\alpha_{k+1}(i)}-\frac{\left(\overline{h_{k+1}(i)}\right)^{2}}{\overline{w_{k+1}(i)}} D_{k}(i) \leq \overline{\alpha_{k+1}(i)}=\sum_{j=1}^{S} \alpha_{k+1}(j) q_{i j}(k)<0 .
$$

By the mathematical induction, the proposition is proved.

Proposition 5 shows that $\alpha_{0}\left(\xi_{0}\right)<0$ for all $\xi_{0} \in \Pi$. By (40), the optimal solution of Problem (41) exists and, by the first-order condition, is given by

$$
\mu^{*}=-\frac{h_{0}\left(\xi_{0}\right) x_{0}+g_{0}\left(\xi_{0}\right) c y_{0}-d}{\alpha_{0}\left(\xi_{0}\right)} .
$$

Plugging (42) into (24) and noting that $x=x_{k}, y=y_{k}$ and $i=\xi_{k}$, we obtain the optimal investment strategy for the mean-variance model (7)

$$
\begin{aligned}
& \boldsymbol{u}_{k}^{*}\left(x_{k}, y_{k}, \xi_{k}\right)=-\mathrm{E}^{-1}\left[\boldsymbol{P}_{k}\left(\xi_{k}\right) \boldsymbol{P}_{k}^{\prime}\left(\xi_{k}\right)\right]\left(\left(x_{k}+c y_{k}\right) \mathrm{E}\left[e_{k}^{0}\left(\xi_{k}\right) \boldsymbol{P}_{k}^{\prime}\left(\xi_{k}\right)\right]+\frac{c y_{k} \overline{\phi_{k+1}\left(\xi_{k}\right)}}{\overline{w_{k+1}\left(\xi_{k}\right)}}\right. \\
& \left.\quad \times \mathrm{E}\left[b_{k}\left(\xi_{k}\right) \boldsymbol{P}_{k}^{\prime}\left(\xi_{k}\right)\right]-\frac{\left(h_{0}\left(\xi_{0}\right) x_{0}+g_{0}\left(\xi_{0}\right) c y_{0}\left(\xi_{0}\right)-d\right) \overline{h_{k+1}\left(\xi_{k}\right)}}{\alpha_{0}\left(\xi_{0}\right) \bar{w}_{k+1}\left(\xi_{k}\right)} \mathrm{E}\left[\boldsymbol{P}_{k}^{\prime}\left(\xi_{k}\right)\right]\right) .
\end{aligned}
$$

Again plugging (42) into (41), we obtain the optimal value of the mean-variance model (7), namely, the minimum variance as follows

$$
\begin{aligned}
& \operatorname{Var}^{*}\left[x_{T^{\tau}}\right]= \\
& \left\{\begin{array}{c}
-\frac{1+\alpha_{0}\left(\xi_{0}\right)}{\alpha_{0}\left(\xi_{0}\right)}\left(d-\frac{h_{0}\left(\xi_{0}\right) x_{0}+g_{0}\left(\xi_{0}\right) c y_{0}}{1+\alpha_{0}\left(\xi_{0}\right)}\right)^{2}+2 \phi_{0}\left(\xi_{0}\right) x_{0} c y_{0} \\
+w_{0}\left(\xi_{0}\right) x_{0}^{2}+\gamma_{0}\left(\xi_{0}\right) c^{2} y_{0}^{2}-\frac{1}{1+\alpha_{0}\left(\xi_{0}\right)}\left(h_{0}\left(\xi_{0}\right) x_{0}+g_{0}\left(\xi_{0}\right) c y_{0}\right)^{2}, \alpha_{0}\left(\xi_{0}\right) \neq-1, \\
-2\left(h_{0}\left(\xi_{0}\right) x_{0}+g_{0}\left(\xi_{0}\right) c y_{0}\right) d+\left(h_{0}\left(\xi_{0}\right) x_{0}+g_{0}\left(\xi_{0}\right) c y_{0}\right)^{2} \\
+w_{0}\left(\xi_{0}\right) x_{0}^{2}+2 \phi_{0}\left(\xi_{0}\right) x_{0} c y_{0}+\gamma_{0}\left(\xi_{0}\right) c^{2} y_{0}^{2}, \quad \alpha_{0}\left(\xi_{0}\right)=-1 .
\end{array}\right.
\end{aligned}
$$

Obviously, according to the definition of variance, for any real number $d$, we must have $\operatorname{Var}^{*}\left[x_{T^{\tau}}\right] \geq 0$. Therefore, we can exclude the case of $\alpha_{0}\left(\xi_{0}\right)=-1$. Then, the minimum variance should be

$$
\begin{aligned}
& \operatorname{Var}^{*}\left[x_{T^{\tau}}\right]=-\frac{1+\alpha_{0}\left(\xi_{0}\right)}{\alpha_{0}\left(\xi_{0}\right)}\left(d-\frac{h_{0}\left(\xi_{0}\right) x_{0}+g_{0}\left(\xi_{0}\right) c y_{0}}{1+\alpha_{0}\left(\xi_{0}\right)}\right)^{2}+w_{0}\left(\xi_{0}\right) x_{0}^{2} \\
& \quad+2 \phi_{0}\left(\xi_{0}\right) x_{0} c y_{0}+\gamma_{0}\left(\xi_{0}\right) c^{2} y_{0}^{2}-\frac{1}{1+\alpha_{0}\left(\xi_{0}\right)}\left(h_{0}\left(\xi_{0}\right) x_{0}+g_{0}\left(\xi_{0}\right) c y_{0}\right)^{2} .
\end{aligned}
$$

Again $\operatorname{Var}^{*}\left[x_{T^{\tau}} \mid\left(z_{0}, \xi_{0}\right)\right] \geq 0$ for any real number $d$ implies $-\frac{1+\alpha_{0}\left(\xi_{0}\right)}{\alpha_{0}\left(\xi_{0}\right)}>0$. Setting $d=d_{\sigma_{\min }}:=\frac{h_{0}\left(\xi_{0}\right) x_{0}+g_{0}\left(\xi_{0}\right) c y_{0}}{1+\alpha_{0}\left(\xi_{0}\right)}$, we obtain the global minimum variance

$$
\begin{aligned}
& \operatorname{Var}_{\text {min }}^{*}\left[x_{T^{\tau}}\right]:=w_{0}\left(\xi_{0}\right) x_{0}^{2}+2 \phi_{0}\left(\xi_{0}\right) c x_{0} y_{0} \\
& \quad+\gamma_{0}\left(\xi_{0}\right) c^{2} y_{0}^{2}-\frac{1}{1+\alpha_{0}\left(\xi_{0}\right)}\left(h_{0}\left(\xi_{0}\right) x_{0}+g_{0}\left(\xi_{0}\right) c y_{0}\right)^{2} .
\end{aligned}
$$

To summarize, we obtain the following results. 
Theorem 2. For given expected terminal wealth $\mathrm{E}\left[x_{T^{\tau}}\right]=d\left(d \geq d_{\sigma_{\min }}\right)$, the efficient investment strategy and the efficient frontier of the multi-period mean-variance $D C$ pension funds investment problem (7) with regime-switching and mortality risk are given by (43) and (45), respectively.

\section{Some special cases}

In this section, we present some special cases of our model.

Special case 1: The case of no pension contribution. This corresponds to $c=0$ in our model. According to (43) and (45), the efficient investment strategy and the efficient frontier are not affected by the state variable $y_{k}$, and are given by

$$
\begin{aligned}
\boldsymbol{u}_{k}^{*}\left(x_{k}, \xi_{k}\right)= & -\mathrm{E}^{-1}\left[\boldsymbol{P}_{k}\left(\xi_{k}\right) \boldsymbol{P}_{k}^{\prime}\left(\xi_{k}\right)\right]\left(x_{k} \mathrm{E}\left[e_{k}^{0}\left(\xi_{k}\right) \boldsymbol{P}_{k}^{\prime}\left(\xi_{k}\right)\right]\right. \\
& \left.-\frac{\left(h_{0}\left(\xi_{0}\right) x_{0}-d\right) \overline{h_{k+1}\left(\xi_{k}\right)}}{\alpha_{0}\left(\xi_{0}\right) w_{k+1}\left(\xi_{k}\right)} \mathrm{E}\left[\boldsymbol{P}_{k}^{\prime}\left(\xi_{k}\right)\right]\right)
\end{aligned}
$$

and

$$
\operatorname{Var}^{*}\left[x_{T^{\tau}}\right]=-\frac{1+\alpha_{0}\left(\xi_{0}\right)}{\alpha_{0}\left(\xi_{0}\right)}\left(d-\frac{h_{0}\left(\xi_{0}\right) x_{0}}{1+\alpha_{0}\left(\xi_{0}\right)}\right)^{2}+\left(w_{0}\left(\xi_{0}\right)-\frac{h_{0}^{2}\left(\xi_{0}\right)}{1+\alpha_{0}\left(\xi_{0}\right)}\right) x_{0}^{2},
$$

respectively, where $w_{k}\left(\xi_{k}\right), h_{k}\left(\xi_{k}\right)$ and $\alpha_{k}\left(\xi_{k}\right)$ are given by (30) and (34).

Special case 2: The terminated time is deterministic. In this case, we only need to let the instantaneous hazard rate $\lambda(s)=0$ over $[0, T]$. Then by $(6)$, we have

$$
p_{i}=0, \quad i=1,2, \cdots, T-1 ; p_{T}=1 \text {. }
$$

The vector series of $\boldsymbol{w}_{k}$ and $\boldsymbol{h}_{k}$ that are directly associated with $p_{i}$ can be simplified as

$$
\boldsymbol{w}_{k}=\left(\prod_{t=k}^{T-1} \operatorname{diag}\left(\boldsymbol{A}_{t}\right) \mathbb{Q}(t)\right) \mathbf{1}, \boldsymbol{h}_{k}=\left(\prod_{t=k}^{T-1} \operatorname{diag}\left(\boldsymbol{J}_{t}\right) \mathbb{Q}(t)\right) \mathbf{1} .
$$

Other vector series of $\boldsymbol{\alpha}_{k}, \boldsymbol{\lambda}_{k}, \boldsymbol{\gamma}_{k}$ and $\boldsymbol{g}_{k}$ are given by (34) and (38). Note that they are related to the series of $\boldsymbol{w}_{k}$ and $\boldsymbol{h}_{k}$, then they are associated indirectly with $p_{i}$. That is to say, their resulting values also change accordingly. As a consequence, the expressions for the efficient investment strategy and the efficient frontier in (43) and (45) can be simplified.

Special case 3: The market environment is deterministic. This means that there is only one state in the market. We let the market state $\Pi$ be a single point set, for example $\Pi=\{1\}$. In this case, we have

$$
\left\{\begin{array}{l}
m=1, \xi_{k} \equiv 1, \mathbb{Q}(k)=q_{11}(k)=1, \overline{w_{k}(1)}=\boldsymbol{w}_{k}=w_{k}(1) \\
\boldsymbol{h}_{k}=\overline{h_{k}(1)}=h_{k}(1), \boldsymbol{\alpha}_{k}=\overline{\alpha_{k}(1)}=\alpha_{k}(1), \boldsymbol{\phi}_{k}=\overline{\phi_{k}(1)}=\phi_{k}(1) \\
\boldsymbol{g}_{k}=\overline{g_{k}(1)}=g_{k}(1), \boldsymbol{\gamma}_{k}=\overline{\gamma_{k}(1)}=\gamma_{k}(1), \quad k=0,1, \cdots, T-1
\end{array}\right.
$$


All the other results in previous sections also hold. Similar to the analysis in Special case 2 , in this case, the computations for all vector series $\boldsymbol{w}_{k}, \boldsymbol{h}_{k}, \boldsymbol{\alpha}_{k}, \boldsymbol{\lambda}_{k}, \boldsymbol{\gamma}_{k}$ and $\boldsymbol{g}_{k}$ would be simplified by (51). Therefore, the computations of the efficient investment strategy and the efficient frontier also can be simplified.

\section{Numerical illustration}

In this section, using real data from the American market, we provide a numerical example to illustrate our results.

Consider a wage earner who enters a DC pension fund plan at time 0 with an initial fund paid $x_{0}=3$ and an initial wage income $y_{0}=1$. Suppose that he/she contribute $20 \%$ of his/her wage income at the beginning of every period, i.e., $c=0.2$. $\mathrm{He} /$ she plans to contribute dynamically for $T=6$ periods and retires at time 6 . Due to the mortality risk, the actual terminated time of his/her pension fund plan is $T^{\tau}$. The probability distribution of $T^{\tau}$ is defined by (6) with the instantaneous hazard rate $\lambda(s) \equiv 0.1$ for $s \in[0, T]$.

Suppose that during the accumulation phase, the pension fund can be invested in the four biggest stocks in the United Sates that have been listed until 2000. These four stocks are MICROSOFT (10107), EXXON MOBIL (11850), GENERAL ELECTRI (12060), WALMART STORES (55976) (labeled by stocks 0, 1, 2, 3). Our data set is composed of the historical monthly return of the four stocks and the U.S.A. average monthly salary from January, 2000, to December, 2013, with sample size 168. Following most literatures (such as Quandt (1958), Chen et al. (2008), Costa and Araujo (2008), Chen and Yang (2011), and Costa and Oliveira (2012)), in this example, the market states are roughly divided into two regimes: $i=1$ is bearish, and $i=2$ is bullish. The states of the Markov chain are classified according to the average growth rate of the four stocks and the salary in a given period (monthly). If the average growth rate in a given period is less than $q_{0.5}$, the state of the Markov chain is said to be in State 1, otherwise, is said to be in to State 2 , where $q_{0.5}$ is the empirical median (based on the above historical data) of the average return. Based on the data set above, we obtain the related parameters for $k=0,1, \cdots, 5$ and different market regimes (states) as follows

$$
\mathrm{E}\left[\boldsymbol{P}_{k}(1) \boldsymbol{P}_{k}^{\prime}(1)\right]=\left(\begin{array}{ccc}
0.0133 & 0.0070 & 0.0078 \\
0.0070 & 0.0153 & 0.0068 \\
0.0078 & 0.0068 & 0.0130
\end{array}\right),
$$




$$
\begin{aligned}
& \mathrm{E}\left[e_{k}^{0}(1)\right]=0.9419, \mathrm{E}\left[b_{k}(1)\right]=1.0025, \mathrm{E}\left[b_{k}^{2}(1)\right]=1.0050, \\
& \mathrm{E}\left[\left(e_{k}^{0}(1)\right)^{2}\right]=0.8949, \mathrm{E}\left[e_{k}^{0}(1) b_{k}(1)\right]=0.9443, \\
& \mathrm{E}\left[\boldsymbol{P}_{k}(1)\right]=(0.0338,0.0036,0.0424)^{\prime}, \\
& \mathrm{E}\left[b_{k}(1) \boldsymbol{P}_{k}(1)\right]=(0.0339,0.0037,0.0425)^{\prime}, \\
& \mathrm{E}\left[e_{k}^{0}(1) \boldsymbol{P}_{k}(1)\right]=(0.0244,-0.0036,0.0323)^{\prime}, \\
& \left.\qquad \begin{array}{ccc}
0.01 & 0.0066 & 0.0068 \\
0.0066 & 0.01 & 0.0058 \\
0.0068 & 0.0058 & 0.0081
\end{array}\right) \\
& \mathrm{E}\left[\boldsymbol{P}_{k}(2) \boldsymbol{P}_{k}^{\prime}(2)\right]=1.0552, \mathrm{E}\left[b_{k}(2)\right]=1.0022, \mathrm{E}\left[b_{k}^{2}(2)\right]=1.0044, \\
& \mathrm{E}\left[\left(e_{k}^{0}(2)\right)^{2}\right]=1.1193, \mathrm{E}\left[e_{k}^{0}(2) b_{k}(2)\right]=1.0576, \\
& \mathrm{E}\left[\boldsymbol{P}_{k}(2)\right]=(-0.0231,-0.0094,-0.0348)^{\prime}, \\
& \mathrm{E}\left[b_{k}(2) \boldsymbol{P}_{k}(2)\right]=(-0.0232,-0.0094,-0.0348)^{\prime}, \\
& \mathrm{E}\left[e_{k}^{0}(2) \boldsymbol{P}_{k}(2)\right]=(-0.0311,-0.0156,-0.0419)^{\prime} .
\end{aligned}
$$

Using the above historical data, we now derive the state transition probability matrix $\mathbb{Q}$ of the Markov chain. According to the classification of the market states for all the historical periods (months), there are $84=168 / 2$ historical periods in state 1. Among all these 84 historical periods being in State 1, we find that the number of the next period in State 1 is 43, and the number of the next period in State 2 is 41 . Therefore, we calculate the empirical state transition probabilities $q_{11}(k)$ and $q_{12}(k)$ as follows

$$
q_{11}(k)=43 / 84 \approx 0.5119, q_{12}(k)=41 / 84 \approx 0.4881 .
$$

Similarly, we can calculate other empirical state transition probabilities $q_{21}(k)=$ $40 / 84=0.4762$ and $q_{22}(k)=44 / 84=0.5238$. Hence the state transition probability matrix is

$$
\mathbb{Q}(k)=\left(\begin{array}{ll}
q_{11}(k) & q_{12}(k) \\
q_{21}(k) & q_{22}(k)
\end{array}\right)=\left(\begin{array}{ll}
0.5119 & 0.4881 \\
0.4762 & 0.5238
\end{array}\right), \quad k=0,1, \cdots, 5 .
$$

Now we present the solving steps for our mean-variance problem (7). Plugging the above data into (43), we obtain the efficient investment strategy for different market states at time 0 and time $k$. For example, when $\xi_{0}=1$ and $\xi_{k}=1$, the efficient 
investment strategy (for stocks $1,2,3$ ) is

$$
\begin{aligned}
& \boldsymbol{u}_{k}^{*}\left(x_{k}, y_{k}, 1\right)=\left(\begin{array}{c}
-1.2672 \\
2.0680 \\
-2.8186
\end{array}\right)\left(x_{k}+0.2 y_{k}\right)+\frac{0.2 y_{k} \overline{\phi_{k+1}(1)}}{\overline{w_{k+1}(1)}} \\
& \times\left(\begin{array}{c}
-1.6349 \\
1.9972 \\
-3.3485
\end{array}\right)+\frac{(1.4888-d) \overline{h_{k+1}(1)}}{0.5988 \overline{w_{k+1}(1)}}\left(\begin{array}{c}
-1.6274 \\
1.9939 \\
-3.3411
\end{array}\right), \quad k=0,1, \cdots, 5,
\end{aligned}
$$

and the efficient investment strategy for stock 0 is $u_{k}^{0}=\left(x_{k}+0.2 y_{k}\right)-\mathbf{1}^{\prime} \boldsymbol{u}_{k}^{*}\left(x_{k}, y_{k}, 1\right)$, where $\mathbf{1}=(1,1,1)^{\prime}$, and

$$
\left\{\begin{array}{l}
\left(\overline{w_{1}(1)}, \cdots, \overline{w_{6}(1)}\right)=(0.5037,0.5004,0.5075,0.5261,0.5580,0.6065), \\
\left.\overline{\phi_{1}(1)}, \cdots, \overline{\phi_{6}(1)}\right)=(1.4956,1.3343,1.1291,0.8585,0.4942,0) \\
\left.\overline{h_{1}(1)}, \cdots, \overline{h_{6}(1)}\right)=(0.4964,0.4940,0.5021,0.5222,0.5560,0.6065) .
\end{array}\right.
$$

When $\xi_{0}=2$ and $\xi_{k}=2$, the efficient investment strategy for stocks $1,2,3$ is

$$
\begin{aligned}
& \boldsymbol{u}_{k}^{*}\left(x_{k}, y_{k}, 2\right)=\left(\begin{array}{c}
0.1042 \\
-2.4634 \\
6.8669
\end{array}\right)\left(x_{k}+0.2 y_{k}\right)+\frac{0.2 y_{k} \overline{\phi_{k+1}(2)}}{\overline{w_{k+1}(2)}} \\
& \times\left(\begin{array}{c}
-0.3285 \\
-2.5277 \\
6.3944
\end{array}\right)+\frac{(1.5703-d) \overline{h_{k+1}(2)}}{0.5994 \overline{w_{k+1}(2)}}\left(\begin{array}{c}
-0.3246 \\
-2.5256 \\
6.3808
\end{array}\right), \quad k=0,1 \cdots, 5,
\end{aligned}
$$

and the efficient investment strategy for stock 0 is $u_{k}^{0}=\left(x_{k}+0.2 y_{k}\right)-\mathbf{1}^{\prime} \boldsymbol{u}_{k}^{*}\left(x_{k}, y_{k}, 2\right)$, where

$$
\left\{\begin{array}{l}
\left(\overline{w_{1}(2)}, \cdots, \overline{w_{6}(2)}\right)=(0.5056,0.5023,0.5094,0.5282,0.5602,0.6065), \\
\left.\overline{\phi_{1}(2)}, \cdots, \overline{\phi_{6}(2)}\right)=(1.4999,1.3383,1.1327,0.8615,0.4964,0) \\
\left.\overline{h_{1}(2)}, \cdots, \overline{h_{6}(2)}\right)=(0.4973,0.4949,0.5031,0.5232,0.5571,0.6065) .
\end{array}\right.
$$

In order to show how the market state would affect the optimal investment strategies, we set $k=3, x_{k}=3.8, y_{k}=1.1$, and plot the optimal investments in these four stocks for different values of mean $d$ (expected terminal wealths) in Figure 1 for both bullish $\left(\xi_{0}=2, \xi_{k}=2\right)$ and bearish $\left(\xi_{0}=1, \xi_{k}=1\right)$ cases. From Figure 1 , we find that, (i) for both stock 0 and stock 2 , the investments increase along with $d$ in bullish market, while, the investments decrease along with $d$ in bearish market, and when $d$ is bigger than $d^{*}$ (the mean corresponding to the intersection 
of the two efficient investment strategies, similarly hereinafter), the bullish market investments are bigger than those in bearish market; (ii) for stock 1, as $d$ increases, the investment increases in both bearish and bullish markets, and when $d$ is bigger than $d^{*}$, the investment in bearish market is bigger than that in bullish market; (iii) for stock 3, as $d$ increases, the investment decreases in bullish market while increases in bearish market, and when $d$ is bigger than $d^{*}$, the investment in bearish market is bigger than that in bullish market. Therefore we could conclude that, no matter in bullish or bearish market, one would invest more in some stocks, but invest less in some other stocks. This is not difficult to understand since the total investable wealth is limited.

We can also present the efficient investment strategy for case $\xi_{0}=2$ and $\xi_{k}=1$, and case $\xi_{0}=1$ and $\xi_{k}=2$. We omit the detailed numerical results here for simplicity.

Plugging the above data into (45), the efficient frontier for each initial state are obtained as follows

$$
\operatorname{Var}\left[x_{T^{\tau}}\right]=\left\{\begin{array}{l}
0.6701(d-3.7105)^{2}+0.1812, \text { when } \xi_{0}=1, \\
0.6682(d-3.9203)^{2}+0.1870, \text { when } \xi_{0}=2,
\end{array}\right.
$$

which are plotted in Figure 2. The efficient frontier is a concept in modern portfolio theory introduced by Markowitz (1952) and others. It is the optimal portfolios plotted along the curve that have the highest expected return for the given amount of risk. Figure 2 illustrates the impact of different starting market states. It is reasonable to start our investment when the market is bullish. In other words, we will expect a higher expected rate of return with lower risk when entering the market at $\xi_{0}=2$. In Figure 2, we observe a higher expected return for a given variance level in the bullish case, which indicates that we would better to enter the market at a bullish time. This observation is consistent with the continuous-time model in Chen et al. (2008).

In the following, we analyze the impacts of some main parameters $c, \lambda(s)$ and $\mathbb{Q}(k)$ on the efficient frontier, assuming that we always enter the market at bearish time, that is, $\xi_{0}=1$. Hereinafter, unless other stated, the related parameters are the same as above.

Figure 3 gives the efficient frontiers corresponding to different contribution rates $c=0,0.1,0.2,0.3,0.4$ and 0.5 . In Figure 2, we find that (i) when $c$ increases from 0 to 0.5 , the efficient frontier moves to the upper right; (ii) the greater $c$ is, the bigger the global minimum variance $\sigma_{\min }^{2}$ as well as its corresponding expected terminal wealth $d_{\sigma_{\min }}$. The result is reasonable since a higher value of $c$ corresponds 

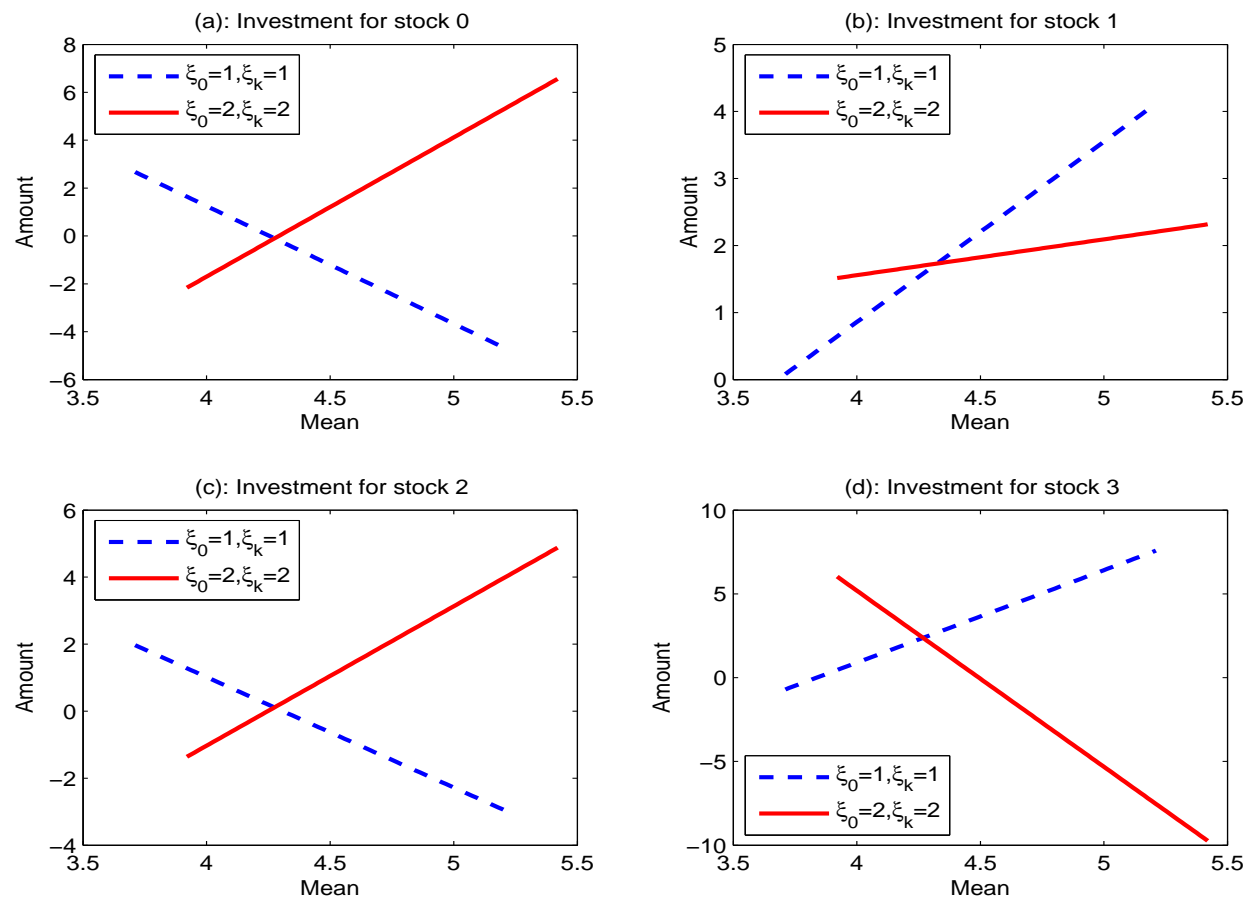

Figure 1: Efficient investment strategies with different market states

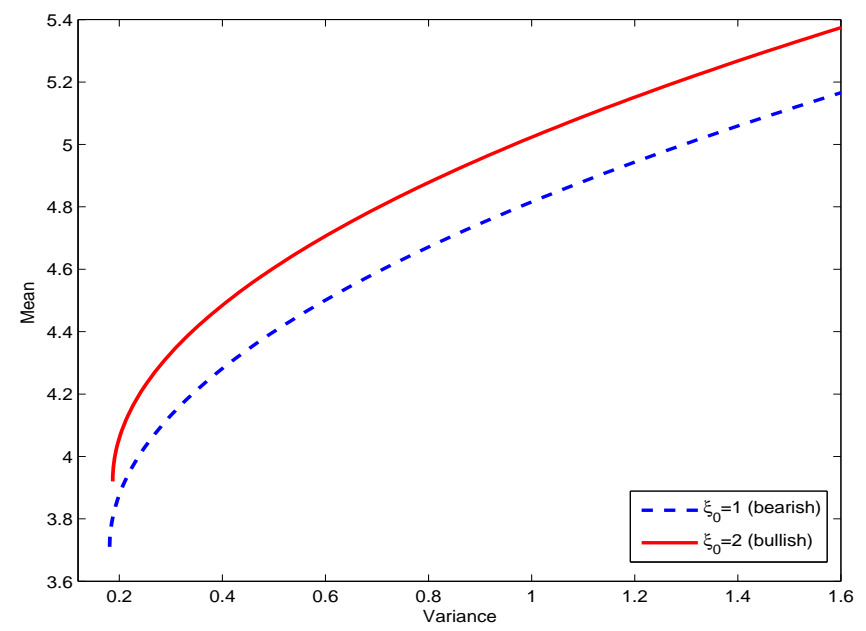

Figure 2: Efficient frontiers with different initial market states 


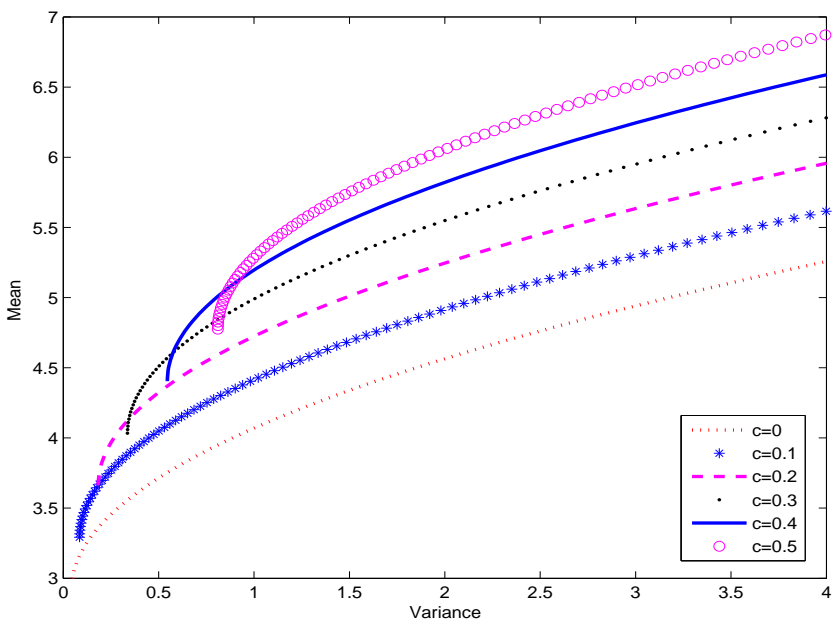

Figure 3: Impact of the contribution rate on the efficient frontier

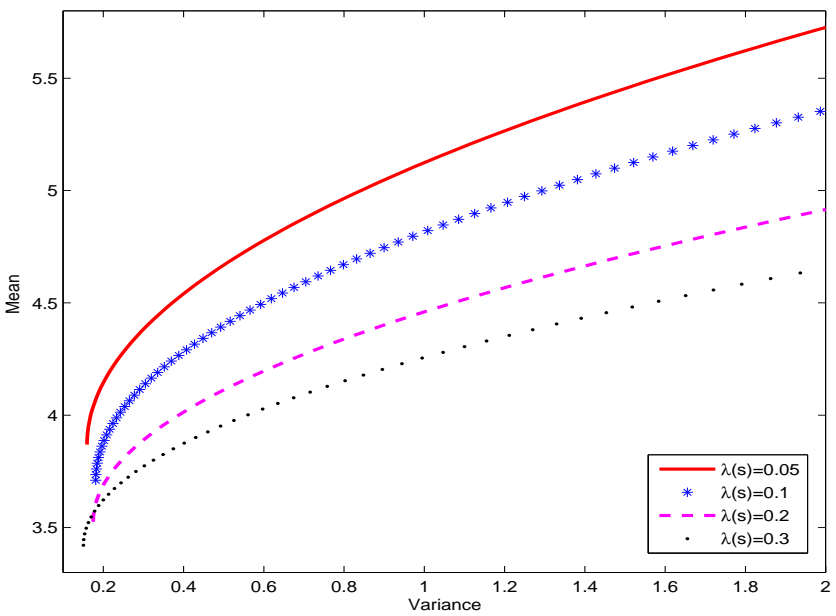

Figure 4: Impact of the instantaneous hazard rate on the efficient frontier 
Table 1: Different state transition probabilities

\begin{tabular}{lllllll}
\hline$q_{11}(k)$ & 0 & 0.2 & 0.4 & 0.6 & 0.8 & 1 \\
$q_{22}(k)$ & 1 & 0.8 & 0.6 & 0.4 & 0.2 & 0 \\
\hline
\end{tabular}

to greater wealth of our pension fund.

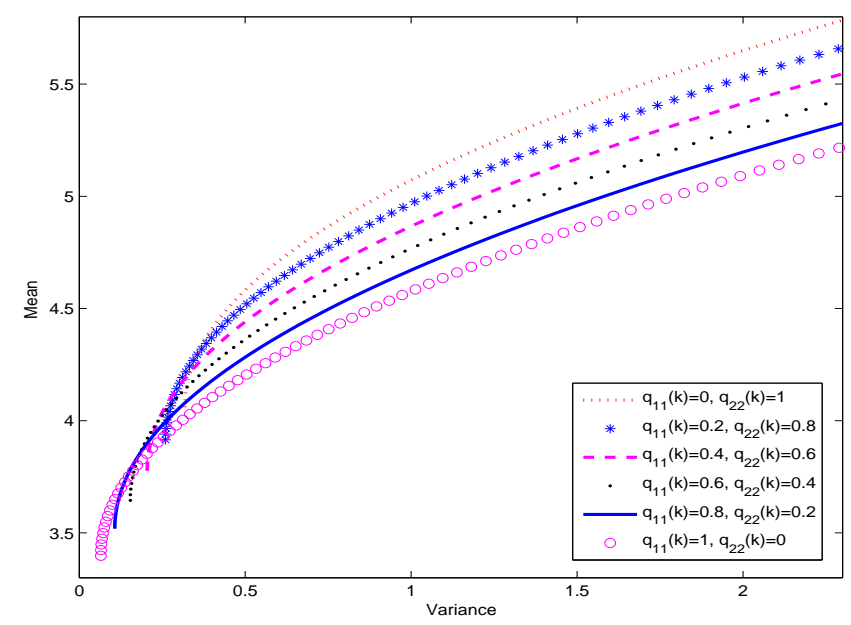

Figure 5: Impact of the state transition probability matrix on the efficient frontier

Figure 4 illustrates the sensitivity of the efficient frontier to the hazard rate $\lambda(s)$. As $\lambda(s)$ increases, the wage earner is more likely to die before the planned time of retirement. This means that the accumulation period for the pension fund is shorter, then we expect a smaller expected terminal wealth for a fixed risk level. Besides, since the overall risk is also smaller for a shorter time period, then we would have a smaller global minimum variance as well as the corresponding expected terminal wealth. We can see those trends in Figure 4 , as $\lambda(s)$ increases from 0.05 to 0.3 , the efficient frontier moves to the lower left.

Finally, we proceed with the impact of the state transition probability $\mathbb{Q}(k)$ on the efficient frontier. Note that $q_{12}(k)=1-q_{11}(k)$ and $q_{21}(k)=1-q_{22}(k)$, we only need to determine the value of $q_{11}(k)$ and $q_{22}(k)$. Different transition probabilities for $q_{11}(k)$ and $q_{22}(k)$ are listed in Table 1 to indicate that the market becomes 'more bearish' when $q_{11}(k)$ increases from 0 to 1 while $q_{22}(k)$ decrease from 1 to 0 . We see from Figure 5 that the corresponding efficient frontier moves to the lower left during this shift. This indicates an decreasing expected rate of return for the same level of investment risk, which is reasonable. 


\section{Conclusion}

In this paper, we adopts the mean-variance criterion to investigate a multi-period DC pension fund portfolio selection problem. To make the model more practical, we incorporate the mortality risk for the wage earner as well as the Markovian regime switching market state. By the Lagrange duality method and the dynamic programming approach, and the matrix representation technique, explicit expressions for the efficient investment strategy and the efficient frontier are derived. We also present some special cases of our model. Finally, a numerical example based on real data from the American market is provided to shed light on the theoretical results established in this paper.

Obviously, a number of problems are worth to be investigated further. For example, we can extend our model to the continuous-time case. Also, we can include a practical constrain such as the bankruptcy constrain or the no-short selling constrain on the investment strategy. Another interesting topic is the robustness of our model. Here all the parameters in our model are determinate and known; in practice, they need to be estimated. The estimation errors can make the optimal solution far from the true optimal solution. Besides, it would also be interesting to study the case where all the parameters are random.

\section{References}

[1] Blake, D., Wright D, Zhang, Y., 2013. Target-driven investing: Optimal investment strategies in defined contribution pension plans under loss aversion. Journal of Economic Dynamics and Control 37, 195-209.

[2] Cairns, A.J.G., Blake, D., Dowd, K., 2006. Stochastic lifestyling: Optimal dynamic asset allocation for defined contribution pension plans. Journal of Economic Dynamics and Control 30, 843-877.

[3] Çanakoglu E., Özekici S., 2010. Portfolio selection in stochastic markets with HARA utility functions. European Journal of Operational Research 201, 520536 .

[4] Chen, P., Yang, H.L., 2011. Markowitz's mean-variance asset-liability management with regime switching: A multi-period model. Applied Mathematical Finance 18, 29-50. 
[5] Chen, P., Yang, H.L., Yin, G., 2008. Markowitz's mean-variance asset-liability management with regime switching: A continuous-time model. Insurance: Mathematics and Economics 43, 456-465.

[6] Chun, C.K., Yang, H.L., 2007. Optimal investment-consumption strategy in a discrete-time model with regime switching. Discrete and Continuous Dynamical Systems-Series B 8, 315-332.

[7] Costa, O.L.V., Araujo, M.V., 2008. A generalized multi-period mean-variance portfolio optimization with Markov switching parameters. Automatica 44, 2487-2497.

[8] Costa, O.L.V., Oliveira, A.D., 2012. Optimal mean-variance control for discretetime linear systems with Markovian jumps and multiplicative noises. Automatica $48,304-315$.

[9] Deelstra, G., Grasselli, M., Koehl, P.F., 2003. Optimal investment strategies in the presence of a minimum guarantee. Insurance: Mathematics and Economics 33, 189-207.

[10] Deelstra, G., Grasselli, M., Koehl, P.F., 2004. Optimal design of the guarantee for defined contribution funds. Journal of Economic Dynamics and Control 28, 2239 - 2260.

[11] Delong, Ł., Gerrard, R., Haberman, S., 2008. Mean-variance optimization problems for an accumulation phase in a defined benefit plan. Insurance: Mathematics and Economics 42, 107-118.

[12] Eichler, M., Türk, D., 2013. Fitting semiparametric Markov regime-switching models to electricity spot prices. Energy Economics 36, 614-624.

[13] Emms, P., 2012. Lifetime investment and consumption using a definedcontribution pension scheme. Journal of Economic Dynamics and Control 36, 1303-1321.

[14] Gao, J.W., 2009. Optimal portfolios for DC pension plans under a CEV model. Insurance: Mathematics and Economics 44, 479-490.

[15] Guan, G.H., Liang, Z.X., 2014. Optimal management of DC pension plan in a stochastic interest rate and stochastic volatility framework. Insurance: Mathematics and Economics 57, 58-66. 
[16] Guan, G.H., Liang, Z., 2015. Mean-variance efficiency of DC pension plan under stochastic interest rate and mean-reverting returns. Insurance: Mathematics and Economics 61, 99-109.

[17] Giacinto, D.M., Federico, S., Gozzi, F., 2011. Pension funds with a minimum guarantee: a stochastic control approach. Finance and Stochastics 15, 297-342.

[18] Haberman, S., Vigna, E., 2002. Optimal investment strategies and risk measures in defined contribution pension schemes. Insurance: Mathematics and Economics 31, 35-69.

[19] Hainaut, D., Devolder, P., 2007. Management of a pension fund under mortality and financial risks. Insurance: Mathematics and Economics 41, 134-155.

[20] Hainaut, D., Deelstra, G., 2011. Optimal funding of defined benefit pension plans. Journal of Pension Economics and Finance 10, 31-52.

[21] Hamilton, J.D., 1989. A new approach to the economic analysis of nonstationary time series and the business cycle. Econometrica 57, 357-384.

[22] Hamilton, J.D., 1994. Susmel R. Autoregressive conditional heteroskedasticity and changes in regime. Journal of Econometrics 64, 307-333.

[23] Han, N.W., Hung, M.W., 2012. Optimal asset allocation for DC pension plans under inflation. Insurance: Mathematics and Economics 51, 172-181.

[24] Hardy, M.R., 2001. A regime-switching model of long-term stock returns. North American Actuarial Journal 5, 41-53.

[25] He, L., Liang, Z.X., 2015. Optimal assets allocation and benefit outgo policies of DC pension plan with compulsory conversion claims. Insurance: Mathematics and Economics 61, 227-234.

[26] Josa-Fombellida, R., Rincón-Zapatero, J.P., 2008. Mean-variance portfolio and contribution selection in stochastic pension funding. European Journal of Operational Research 187, 120-137.

[27] Kim, C.J., Piger, J., Startz, R., 2008. Estimation of Markov regime-switching regression models with endogenous switching. Journal of Econometrics 143, 263-273. 
[28] Korn, R., Siu, T.K., Zhang, A., 2011. Asset allocation for a DC pension fund under regime switching environment. European Actuarial Journal (S2), S361S377.

[29] Konicz, A.K., Mulvey, J., 2015. Optimal savings management for individuals with defined contribution pension plans. European Journal of Operational Research 243, 233-247.

[30] Li, D., Ng, W.L., 2000. Optimal dynamic portfolio selection: Multiperiod meanvariance formulation. Mathematical Finance 10, 387-406.

[31] Liu, H., 2011. Dynamic portfolio choice under ambiguity and regime switching mean returns. Journal of Economic Dynamics and Control 35, 623-640.

[32] Luenberger, D.G., 1968. Optimization by Vector Space Methods. Wiley, New York.

[33] Markowitz, H., 1952. Portfolio selection. Journal of Finance 7, 77-91.

[34] Pliska, S.R., Ye, J.C., 2007. Optimal life insurance purchase and consumption/investment under uncertain lifetime. Journal of Banking \& Finance 31, 1307-1319.

[35] Quandt, R.E., 1958. The Estimation of the Parameters of a linear regression system obeying two separate regimes. Journal of the American Statistical Association $53,873-880$.

[36] Richard, S.F., 1975. Optimal consumption, portfolio and life insurance rules for an uncertain lived individual in a continuous time model. Journal of Financial Economics 2, 187-223.

[37] Shen, Y., Siu, T.K., 2012. Asset allocation under stochastic interest rate with regime switching. Economic Modelling, 2012, 29: 1126-1136.

[38] Siu, T.K., 2012. A BSDE approach to risk-based asset allocation of pension funds with regime switching. Annals of Operations Research 201, 449-473.

[39] Vigna, E., 2014. On efficiency of mean-variance based portfolio selection in defined contribution pension schemes. Quantitative Finance 14, 237-258.

[40] Wu, H.L., Zeng, Y., 2015. Equilibrium investment strategy for definedcontribution pension schemes with generalized mean-variance criterion and mortality risk. Insurance: Mathematics and Economics 64, 396-408. 
[41] Wu, H.L., Zhang, L., Chen, H., 2015. Nash equilibrium strategies for a defined contribution pension management. Insurance: Mathematics and Economics 62, 202-214.

[42] Yaari, M.E., 1965. Uncertain lifetime, life insurance, and the theory of the consumer. Review of Economic Studies 32, 137-150.

[43] Yao, H.X., Yang, Z., Chen, P., 2013. Markowitz's mean-variance defined contribution pension fund management under inflation: a continuous-time model. Insurance: Mathematics and Economics 53, 851-863.

[44] Yao, H.X., Lai, Y.Z., Ma, Q.H., Jian, M.J., 2014. Asset allocation for a DC pension fund with stochastic income and mortality risk: A multi-period meanvariance framework. Insurance: Mathematics and Economics 54, 84-92.

[45] Yiu, K.F.C., Liu, J.Z., Siu, T.K., Ching, W.K., 2010. Optimal portfolios with regime switching and value-at-risk constraint. Automatica 46, 979-989.

[46] Zhou, X.Y., Li, D., 2000. Continuous-time mean-variance portfolio selection: A stochastic LQ framework. Applied Mathematics and Optimization 42, 19-33. 


\section{University Library}

\section{- M M N E R VA A gateway to Melbourne's research publications}

Minerva Access is the Institutional Repository of The University of Melbourne

Author/s:

Yao, $\mathrm{H}$;Chen, $\mathrm{P} ; \mathrm{Li}, \mathrm{X}$

Title:

Multi-period defined contribution pension funds investment management with regimeswitching and mortality risk

\section{Date:}

2016-11-01

\section{Citation:}

Yao, H., Chen, P. \& Li, X. (2016). Multi-period defined contribution pension funds investment management with regime-switching and mortality risk. Insurance: Mathematics and Economics, 71, pp.103-113. https://doi.org/10.1016/j.insmatheco.2016.08.005.

Persistent Link:

http://hdl.handle.net/11343/118411 